\title{
Role of the mobilome in the global dissemination of the carbapenem resistance gene blanDM
}

Mislav Acman ${ }^{1 *}$, Ruobing Wang ${ }^{2}$, Lucy van Dorp ${ }^{1}$, Liam P. Shaw ${ }^{3}$, Qi Wang ${ }^{2}$, Nina Luhmann ${ }^{4}$, Yuyao Yin²,

51 UCL Genetics Institute, University College London, Gower Street, London, WC1E 6BT, UK

62 Department of Clinical Laboratory, Peking University People's Hospital, Beijing, 100044, China

73 Department of Zoology, University of Oxford, Oxford OX1 3SZ, UK

84 Warwick Medical School, University of Warwick, Coventry CV4 7AL, UK

$9 *$ Corresponding Author

10 E-mail: mislav.acman.17@ucl.ac.uk 


\section{Abstract (240 words)}

12 The mobile resistance gene bla $a_{\mathrm{NDM}}$ encodes the NDM enzyme which hydrolyses carbapenems, a class of 13 antibiotics used to treat some of the most severe bacterial infections. bla $a_{\mathrm{NDM}}$ is globally distributed across a variety 14 of Gram-negative bacteria on multiple plasmids, typically located within a highly recombining and transposon15 rich genomic region. This complexity means the dynamics underlying the dissemination of bla $a_{\mathrm{NDM}}$ remain poorly 16 resolved. In this work, we compile a dataset of over 6000 bacterial genomes harbouring the bla $a_{\text {NDM }}$ gene including 17104 newly generated PacBio hybrid assemblies from clinical and livestock associated isolates across China. We 18 develop a novel computational approach to track structural variants surrounding bla NDM $_{\text {in }}$ bacterial genomes.

19 This allows us to identify the prevalent genomic contexts of bla $a_{\mathrm{NDM}}$ and reconstruct the key mobile genetic 20 elements and events in its global spread. We estimate that bla $a_{\mathrm{NDM}}$ emerged on a $\mathrm{Tn} 125$ transposon before 1985 21 but only reached a global prevalence around a decade after its first recorded observation in 2005 . We find that the 22 Tn125 transposon played an important role in early plasmid-mediated jumps of bla NDM $_{\text {but }}$ was overtaken by other 23 elements in recent years including IS26-flanked pseudo-composite transposons and Tn3000. Lastly, we observe 24 a notable correlation between plasmid backbones bearing bla $a_{\mathrm{NDM}}$ and the sampling location of isolates. This 25 observation suggests that the dissemination of resistance genes is mainly driven by successive between-plasmid 26 transposon jumps, with plasmid exchange much more restricted due to the adaptation of plasmids to specific 27 bacterial hosts. 


\section{Introduction}

29 Antimicrobial resistance (AMR) poses a major challenge to human and veterinary health. AMR can be conferred

30 by vertically inherited point mutations or via the acquisition of horizontally transmitted 'accessory' genes, often

31 located in transposons and plasmids. The bla $a_{\mathrm{NDM}}$ gene represents a typical example of a mobile AMR gene'.

32 bla

33 used as a first-line treatment for severe infections and to treat multidrug-resistant Gram-negative bacterial

34 infections. As such, the global prevalence of bacteria carrying bla $a_{\mathrm{NDM}}$ represents a major public health concern.

bla NDM $_{\text {was }}$ first described in 2008 from a Klebsiella pneumoniae isolated from a urinary tract infection in a Swedish patient returning from New Delhi, India ${ }^{2}$. While bla earliest cases have been linked to the Indian subcontinent, leading to this region being suggested as the likely location for the initial mobilisation event ${ }^{1,3-6}$. NDM-positive Acinetobacter baumannii isolates have been retrospectively identified from an Indian hospital in $2005^{7}$, which remain the earliest observations to date. Nevertheless, an NDM-positive A. pittii isolate was also isolated in 2006 from a Turkish patient with no history of travel outside Turkey ${ }^{8}$.

Although no complete genome sequences are publicly available from these earliest observations, the first NDMpositive isolates from 2005 were shown to carry bla NDM on multiple non-conjugative, but potentially mobilizable plasmid backbones ${ }^{7}$. In addition, bla NDM $_{\text {in }}$ these early isolates was positioned within a complete $\mathrm{Tn} 125$ transposon with IS26 insertion sequences (ISs) as well as ISCR27 (IS-containing common region 27), suggesting the possibility of complex patterns of mobility since the gene's initial integration. Subsequent NDM-positive isolates across multiple species consistently harbour either a complete or fragmented ISAba125 (an IS constituting Tn125), immediately upstream of bla $a_{\mathrm{NDM}}$, which provides a promoter region ${ }^{1,5,9,10}$. The presence of ISAba125 in some form in all NDM-positive isolates to date and the early observations in A. baumannii have led to $\operatorname{Tn} 125$ being proposed as the ancestral transposon responsible for the mobilization of bla $a_{\mathrm{NDM}}$, and A. baumannii as the ancestral host $^{10,11}$.

The NDM enzyme itself is of possible chimeric origin ${ }^{10,12}$, with the first six amino acids in NDM matching to those in aphA6, a gene providing aminoglycoside resistance and also flanked by ISAba125. It is hypothesised that ISCR 27, which uses a rolling-circle (RC) transposition mechanism ${ }^{13,14}$, initially mobilized a progenitor of bla NDM in Xanthomonas sp. and placed it downstream of ISAba12510,12,15,16. At least 29 distinct sequence variants of the NDM enzyme have been described to date ${ }^{1,17}$. The most prevalent of these variants is the first to have been characterised, denoted NDM- ${ }^{18}$. Different NDM variants are mostly distinguished by a single amino-acid substitution, apart from NDM-18 which carries a tandem repeat of five amino acids. None of the observed substitutions occur in the active site and their functional impact remains under debate ${ }^{1}$.

bla $\mathrm{NDM}_{\mathrm{N}}$ is found in at least 11 bacterial families and NDM-positive isolates have heterogeneous clonal backgrounds, supporting multiple independent acquisitions of bla $\mathrm{NDM}^{1}$. Although bla ${ }_{\mathrm{NDM}}$ has been observed on bacterial chromosomes ${ }^{19,20}$ it is most commonly found on plasmids, comprising multiple different backbones or types. Thus far, bla 
65 plasmid type, bla $a_{\mathrm{NDM}}$ can be found in a variety of genomic contexts, often interspersed by multiple ISs and 66 composite transposons ${ }^{1,12}$. The immediate environment of bla $a_{\mathrm{NDM}}$ has been reported to vary even in isolates from 67 the same patient ${ }^{23}$. Many mobile elements are thought to play important roles in dissemination, including 68 ISAba125, IS3000, IS26, IS5, ISCR1, Tn3, Tn125, and Tn30001,23,25-28. It is therefore clear that the spread of 69 bla $a_{\mathrm{NDM}}$ was, and is, a multi-layer process involving multiple mobile genetic elements - 'the mobilome'. bla $a_{\mathrm{NDM}}$ 70 mobility involves diverse processes, including genetic recombination ${ }^{29,30}$, transposition, conjugation and 71 transformation of plasmids ${ }^{31}$, transduction ${ }^{32}$, and transfer through outer-membrane vesicles (OMVs) ${ }^{33,34}$.

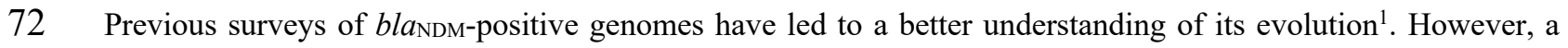
73 major difficulty, as for other AMR genes, is relating the diverse genomic contexts to temporal evolution. Here, 74 we outline an alignment-based method to identify flanking structural variants and use it to build a history of the 75 insertion and mobilization events. We compile a global dataset of more than 6000 NDM-positive isolates. In line 76 with previous studies, we identify Tn125, IS26 and Tn3000 as the main contributors to bla $a_{\mathrm{NDM}}$ mobility but go 77 further and estimate the timing of the initial emergence of bla $a_{\mathrm{NDM}}$ to pre-1990, around two decades prior to its 78 first detection and rapid dissemination. Our findings suggest that this global spread was driven primarily by transposons, with plasmids playing more of a role in local transmission. 


\section{Results}

\section{A global dataset of blanDM carriers}

82 We compiled a dataset of 6155 bacterial genomes (7148 contigs) carrying at least one copy of bla NDM $_{\text {(Figure } 1) .}$ 83 These include: published assemblies from NCBI RefSeq ${ }^{35}(n=2632)$, NCBI GenBank $^{36}(n=1158)$ and Enterobase ${ }^{37}$ 84 ( $n=1379)$; bacterial genomes assembled using short read de novo assembly from NCBI's Sequence Read Archive 85 (SRA) ( $n=882)$; and newly generated bacterial genomes isolated from 79 hospitalized patients across China and 8625 livestock farms assembled using hybrid PacBio-Illumina de novo assembly ( $n=104)$ (Supplementary Table 1, 87 Supplementary Figure 1). While public genomes have inherent sampling biases, using them is the most 88 comprehensive approach available ${ }^{1}$. Data was included from 251 different Bioprojects, with more than half the 89 samples linked to two large-scale database refinement efforts ${ }^{38,39}$.

90 The dataset included bla $a_{\mathrm{NDM}}$-positive isolates from 88 states (Figure 1A) mostly collected in Asia, particularly 91 mainland China ( $n=1270)$, European countries (941), USA (461), Thailand (419) and India (361). At least 27 92 bacterial genera were represented, with a large fraction of Klebsiella and Escherichia isolates (2664 and 2154 genomes respectively; Figure 1B; Supplementary Data 1). Collection dates were recorded for 4816 samples (78.25\%). Of these, the majority were collected between 2014-2019 (71.05\%, Figure 1C). The dataset also includes 55 genomes collected in 2010 or earlier. These include the K. pneumoniae isolate from 2008 Sweden in which bla $_{\mathrm{NDM}}$ was first characterized ${ }^{2}$; one Enterobacter hormaechei isolate from 2008 India $^{40}$; one S. enterica isolate from 2008 London, $\mathrm{UK}^{41}$; one A. baumannii isolate from an individual of Balkan origin collected in Germany in 200722,43; and nine assembled E. coli genomes from urine samples collected in Greece in 2007 (Supplementary Data 1).

100 The dataset contained 17 known variants of NDM. NDM-1 was the most abundant ( $n=4127$; Supplementary 101 Figure 2A) with NDM-5 ( $n=2394)$ increasing in prevalence after 2012 (Supplementary Figure 2B and C). Variants

102 showed different associations with plasmid types (Supplementary Figure 2D) and genera (Supplementary Figure

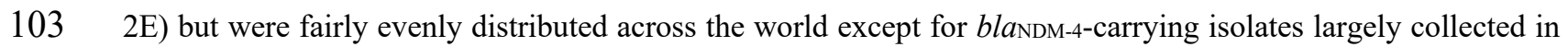

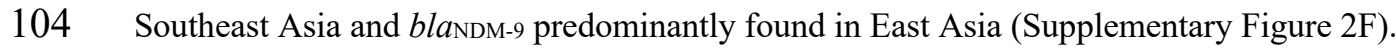




\section{Plasmid backbones carrying blandM}

106 We identified 33 different replicon types on 1222 contigs using PlasmidFinder ${ }^{44}$ (Figure 1D). The most prevalent

107 replicon type was IncX3 (444 contigs), and abundant types exhibited geographic structure (Supplementary Figure

108 3). To further identify uncharacterised plasmid types, we mapped 3599 contigs to a set of complete plasmid 109 reference sequences after discarding short contigs (see Methods). This revealed 181 clusters of similar putative

110 plasmid sequences (Supplementary Figure 4; Supplementary Data 2). Most clusters ( $n=105)$ grouped contigs of 111 the same replicon type and contained a small number of contigs (only 27 clusters included $>10$ contigs), in line 112 with a diverse and dynamic population of plasmid backbones for bla $a_{\mathrm{NDM}}$.

113 The majority $(\mathrm{n}=2427 ; 68.4 \%)$ of bla $\mathrm{NDM}_{\mathrm{ND}}$-carrying contigs were associated with small putative plasmids $(<10 \mathrm{~Kb}$;

114 Supplementary Figure 4). While this could suggest small plasmids play a key role as bla $a_{\mathrm{NDM}}$ carriers, this pattern 115 could also result from consistently fragmented de novo assemblies due to duplicated ISs and transposons. 116 Consistent with this latter hypothesis, 610 contigs mapped to pKP-YQ12450 which is likely a $7.8 \mathrm{~Kb}$ fragment of

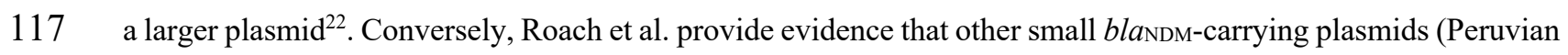
118 pKP-NDM-1_isoforms 1-5) are inherited by descent and are result of transposon-mediated plasmid fusion ${ }^{45}$.

\section{Resolving structural variants in the bla}

120 To go beyond a static reference-based view of variation around bla $a_{\mathrm{NDM}}$ and gain a detailed overview of the possible 121 events in its evolution, we developed an alignment-based approach to progressively resolve genomic variation 122 moving upstream or downstream from the gene (see Methods, Figure 2). In brief, a pairwise discontiguous Mega 123 BLAST search (v2.10.1+ $)^{46,47}$ is applied to all bla $a_{\mathrm{NDM}}$-carrying contigs to identify all possible homologous regions 124 between each contig pair. Only BLAST hits covering the complete bla NDM gene are retained (Figure 2A). Next,

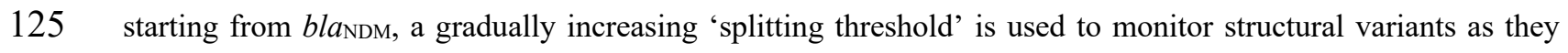
126 appeared upstream or downstream of the gene. At each step, a network of contigs (nodes) that share a BLAST hit 127 with a minimum length as given by the 'splitting threshold' is assessed (Figure 2B). As we move upstream or 128 downstream and further away from the gene, the network starts to split into smaller clusters, each carrying contigs 129 that share an uninterrupted stretch of homologous DNA, which can be represented as a tree (Figure 2C). This 130 approach treats the upstream and downstream flanking regions separately rather than simultaneously and is 131 agnostic to whether splitting into 'sequence clusters' is caused by structural variants of the same genomic 132 background or different genomic backgrounds.

133 Upstream of bla $a_{\mathrm{NDM}},>98 \%$ of sufficiently long contigs included a $\sim 75$ bp fraction of ISAba 125 , supporting Tn 125 134 as an ancestral transposon of the bla $a_{\mathrm{NDM}}$ gene in agreement with previous work ${ }^{1,5,9,10}$ (Supplementary Figures 5 135 and 6). However, the homology of the region upstream of bla $a_{\mathrm{NDM}}$ falls quickly: within a few hundred base pairs

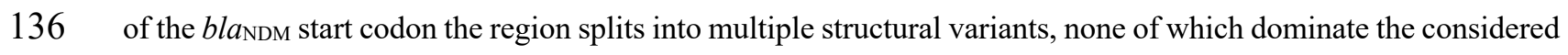
137 pool of contigs (Supplementary Figures 5 and 6). We identified 141 different structural variants within $1200 \mathrm{bp}$ 138 upstream of bla $a_{\mathrm{NDM}}$. This upstream region contained a high number of ISs (e.g. ISAba1 25 [ $n=243$ ], IS5 [ $n=426$ ], 139 IS3000 [ $n=60]$, ISKpn14 [ $n=55]$, and ISEc33 [ $n=147])$. This transposition hotspot probably contributes to 140 fragmented assemblies: 2269 contigs were excluded from further analysis for being too short (Supplementary 141 Figure 3). 
142 The downstream flanking region exhibits more gradual structural diversification than the upstream region, with

143 one dominant putative ancestral background (Figure 3). As illustrated by the stem of the tree of structural variants, 144 many of the 7014 contigs analysed contained complete sequences of the same set of genes: ble (6863 contigs),

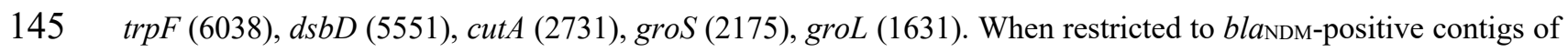
146 sufficient length to possibly harbour the full repertoire of these genes $(n=3786)$, almost half carry all of them $147(n=1,631 ; 43.1 \%)$. In addition, we find dominant structural variants associated with various source databases and 148 sequence lengths hence diminishing the impact of the sampling bias (Supplementary Figure 7)

\section{Early events in the spread of blanDM}

150 While we did not observe any strong overall signal in the distribution of associated plasmid backbones, bacterial 151 genera, or sampling locations, closer examination of mobilome features common to sufficiently large numbers of

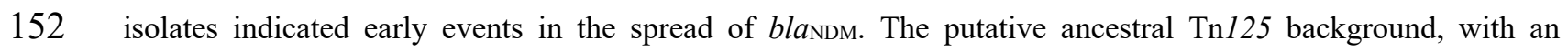
153 uninterrupted downstream ISAba125 element, was seen in contigs mainly from Acinetobacter and Klebsiella 154 (Figure 3 top). Conversely, the diversity of bacterial genera carrying ISAba125 upstream is more substantial

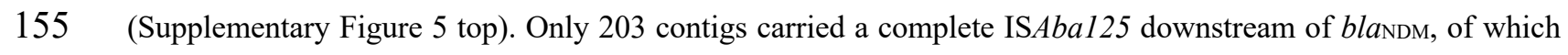
156147 carried an ISAba125 sequence in proximity $(<9 \mathrm{~Kb})$ to the bla $a_{\mathrm{NDM}}$ start codon. These account for a minority $157(7 \%$; 147/2097) of isolates when sufficiently long contigs are considered. This supports the initial dissemination 158 of bla $a_{\mathrm{NDM}}$ by $\mathrm{Tn} 125$ to other plasmid backbones predominately being mediated by Acinetobacter and Klebsiella, 159 after which the transposon was disrupted by other rearrangements.

160 IS3000, both upstream and downstream, was almost exclusively associated with samples from Klebsiella (Figure 1613 and Supplementary Figure 5). Thus, as suggested by Campos et al. ${ }^{26}$, Tn 3000 - a composite transposon made 162 of two copies of IS3000 - likely re-mobilized bla $a_{\mathrm{NDM}}$ following the 'fossilization' of Tn125; our findings suggest 163 this secondary mobilization primarily happened in Klebsiella species. Tn5403 was found extensively associated 164 with IncN2 plasmids (Figure 3) which could have placed bla $a_{\mathrm{NDM}}$ in this background via cointegrate intermediate 165 as previously suggested by Poirel et. al. ${ }^{9}$ Some elements of the mobilome were geographically linked e.g., IS5 166 which was predominantly found upstream of bla $a_{\mathrm{NDM}}$ on IncX3 plasmids in Escherichia from East Asia 167 (Supplementary Figure 5). IS5 is known to enhance transcription of nearby promoters in $E$. coli ${ }^{48}$ and its 168 abundance and positioning just upstream of bla $a_{\mathrm{NDM}}$ suggests a similar role in this case.

169 One of the most commonly identified transposable elements in the downstream flanking region $(\sim 30 \%$ prevalence $)$ 170 was ISCR1 (IS91 family transposase) (Figure 3) always accompanied by sul1 and occasionally in configuration 171 with ant 1 or $p s p$, ampR, and dap genes. In some cases, a small and possibly fragmented putative IS, which we 172 refer to as 'IS-?', is found further downstream. IS-? bears little similarity to known ISs and it is unclear what role 173 it plays in the mobility of bla $a_{\mathrm{NDM}}$. ISCR1 is found at various positions downstream of bla $a_{\mathrm{NDM}}$ and often in 174 Escherichia and Klebsiella species. We note that, in most cases, the orientation of ISCR1 should prevent this 175 element from mobilizing bla $a_{\mathrm{NDM}}$ (Figure 3$)^{14}$. Nevertheless, the prevalence of this element could be due to the 176 several AMR genes it can mobilize, such as sull or ampR. ISCR1s are mainly found in complex class 1 integrons ${ }^{14}$, 177 however, not many annotated integrase genes are located within the vicinity of bla $a_{\mathrm{NDM}}$. In fact, only 15 contigs 178 were found to have an integrase $<50 \mathrm{~Kb}$ away from bla $a_{\mathrm{NDM}}$ and none showed any consistency in integrase 179 placement with respect to bla $\mathrm{NDM}_{\mathrm{N}}$. This suggests integrases play a minor role in the dissemination of $b l a_{\mathrm{NDM}}$. 
180 Another notable ISCR element is ISCR27 which is consistently found immediately downstream of the groL gene

181 at high prevalence (33.1\% of sufficiently long contigs; Figure 3 ). Contrary to its ISCR1 relative, ISCR27 is

182 correctly oriented to mobilize $b l_{\mathrm{NDM}}$ as is presumed to have happened during the initial mobilization of the

183 progenitor of bla $_{\mathrm{NDM}}{ }^{10}$. However, we find no evidence of subsequent ISCR27 mobility. The origin of rolling-circle

184 replication of ISCR27 (oriIS; GCGGTTGAACTTCCTATACC) is located 236 bp downstream of the ISCR27

185 transposase stop codon. The region downstream of this stop codon in all structural variants bearing a complete

186 ISCR27 is highly conserved for at least $750 \mathrm{bp}$ (Figure 3).

\section{Subsequent rearrangements dominated by IS26}

188 Three sharp drops in the number of considered contigs at particular distances downstream of bla $a_{\text {NDM }}$ (see Figure

189 3, e.g., region 3000-3300bp) prompted us to investigate these distinct cut-offs. We mapped 781 raw Illumina

190 paired-end sequencing reads from our dataset back to their matching bla $a_{\mathrm{NDM}}$ contigs. The read overhangs ( $\left.\geq 50 \mathrm{bp}\right)$

191 that mapped to the downstream end of the contigs were screened against the ISFinder database ${ }^{49}$. The $\geq 50 \mathrm{bp}$

192 overhangs associated with 3000-3300 long flanks downstream of bla $a_{\mathrm{NDM}}$ corresponding to the largest observed

193 drop almost exclusively match the left inverted repeat (IRL) of the IS26 sequence (Supplementary Figure 8).

194 Another hotspot, associated to IS26 was found around 7,500bp, while at around 7,800bp a number of overhanging

195 reads mapped to ISAba125. These positions roughly match the third drop in the number of contigs observed 7500-

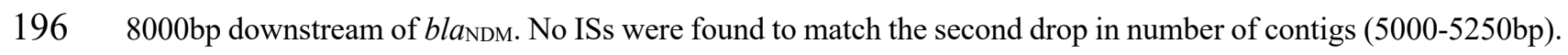

197 IS26, although often found in two adjacent copies forming a seemingly composite transposon, is a so-called 198 pseudo-composite (or pseudo-compound) transposon ${ }^{50}$. In contrast to composite transposons, a fraction of DNA

199 flanked by the two IS26 is mobilized either via cointegrate formation or in the form of a circular translocatable

200 unit (TU), which consists of a single IS26 element and a mobilized fraction of DNA, and inserts preferentially 201 next to another IS $26^{50,51}$. Taken together, the presented results, including Supplementary Figure 8, suggest three

202 possible explanations for the presence of short bla $a_{\mathrm{NDM}}$ carrying contigs in the dataset: (i) the presence of IS26 TUs 203 in the host cell; (ii) other circular DNA formations mediated by plasmid recombination, transposons ${ }^{9,45}$ or ISCR $^{2}$ 204 elements ${ }^{13,52}$; (iii) missasembly of contigs due to presence of multiple copies of the same ISs ${ }^{53}$.

205 To further investigate the mobility of bla $a_{\mathrm{NDM}}$, we characterised the most common (pseudo-)composite transposons

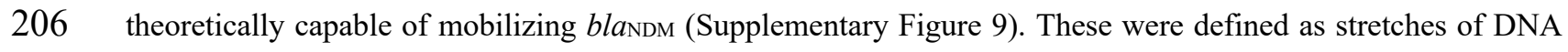
207 flanked by two matching complete or partial ISs $<30 \mathrm{~Kb}$ apart and enclosing bla $a_{\mathrm{NDM}}$. In total, we identified 640 208 composite transposons in 468 contigs which comprised 31 different types with the most frequent being: IS26 (231 209 instances), IS3000 (forming Tn3000; 168), ISAba125 (forming Tn125; 138 instances), and IS15 (28) 210 (Supplementary Figure 9B). Interestingly, we observe 80 cases where $>2$ of the same IS flank bla $a_{\mathrm{NDM}}$. These are 211 mostly IS26 (59) which could indicate the presence of cointegrate formation ${ }^{50}$ and showcases increased activity 212 of this particular insertion element. Only 431 of the 640 putative composite transposons identified contained both 213 complete flanking ISs, while others had at least one IS partially truncated. In addition, 1681 ISCR27, and 150 214 ISCR1 were found in similar proximity and appropriate orientation to mobilize bla $a_{\text {NDM }}$ (Supplementary Figure 215 9B). However, as mentioned earlier, their role in transposition of bla $a_{\mathrm{NDM}}$ appears minor. 
216 In the majority of cases, composite transposons $\operatorname{Tn} 125$ and $\operatorname{Tn} 3000$ were found to have a consistent length ranging

217 from 7-10Kb (Supplementary Figure 9A). Similarly, ISCR1 and ISCR27 are found at fixed positions downstream

218 of bla $a_{\mathrm{NDM}}$. However, the lengths of transposons formed by IS15, a known variant of IS26 $6^{54}$, and especially IS26

219 were found to be more variable. Pairs of IS26 are found to be $2.5-30 \mathrm{~Kb}$ apart again consistent with increased

220 activity and multiple independent insertions. We note that IS15 and IS26 occur at increased presence in samples

221 collected in East and Southeast Asia (Supplementary Figure 9C). These occur roughly equally in Escherichia and

222 Klebsiella genera (Supplementary Figure 9D) and are associated to multiple plasmid backbones, but

223 predominantly on IncF plasmids (Supplementary Figure 9E). Tn125 and Tn3000 have a notable predominance in

224 the Indian subcontinent (Supplementary Figure 9C) and largely in the Acinetobacter and Klebsiella genera

225 respectively (Supplementary Figure 9D).

\section{Molecular dating of key events}

227 We estimated the relative timing of the formation of the $\mathrm{Tn} 125$ and $\mathrm{Tn} 3000$ transposons (see Methods). After

228 selecting only contigs with conserved transposon configurations we aligned each transposon region and identified

229 the likely root (i.e., ancestral) sequence by assessing temporal patterns (Supplementary Figures 10 and 11; see

230 Methods). Overall, we observed fewer SNPs, mostly located within the transposase gene, in the alignment of

231 Tn3000 compared to Tn125, but observed a significant temporal signal for both (Supplementary Figures 12-13).

232 We also assessed temporal signal for three other prevalent insertion events (Figure 3), namely: bla $a_{\mathrm{NDM}}$ with

233 downstream ISCR27, bla $a_{\mathrm{NDM}}$ with correctly oriented downstream folP-ISCR1 (+ strand), and bla $a_{\mathrm{NDM}}-\mathrm{dsbD}$ with

234 downstream ISCR1 (- strand) ending with an unknown putative IS (labelled IS-?). However, no significant

235 temporal signal was recovered for these events.

236 This Bayesian analysis indicated that the most recent common ancestor (MRCA) of the Tn125 transposon carrying 237 the bla $a_{\mathrm{NDM}}$ gene dated to before 1990 (Figure 4A). While the time intervals are uncertain, the results are consistent

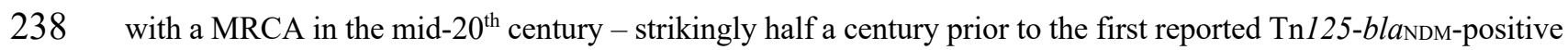
239 isolates ${ }^{7}$. Conversely, the mobilization of bla $a_{\mathrm{NDM}}$ by $\mathrm{Tn} 3000$ is estimated to have happened later at the turn of the 240 millennium (Figure 4B). These findings are consistent with a wider narrative whereby the spread of bla $a_{\mathrm{NDM}}$ was 241 initially driven by Tn 125 mobilization before subsequent transposition by Tn3000, IS 26 and others.

\section{Temporal diversity in blanDM isolates suggests role of plasmids}

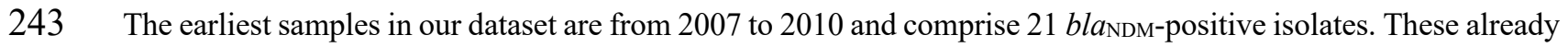

244 encompass seven bacterial species, collected in eight countries spanning four geographic regions (17 clinical 245 samples and four of unknown origin from South Asia, Middle East, Oceania, and Europe). Such a wide host and 246 geographic distribution, even in the earliest available genomes, illustrates the extraordinarily high mobility of

$247 b l a \mathrm{NDM}$ at this stage and is consistent with our molecular dating estimates.

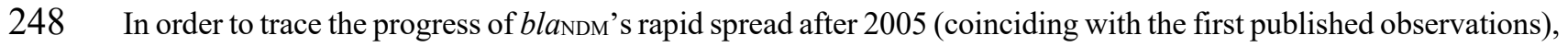

249 we measured diversity over time for several metadata categories, including country, genera, plasmid backbone 250 and IS presence (Supplementary Figure 16; see Methods). The change in diversity of the countries associated to $251 b l a_{\mathrm{NDM}}$-positive isolates was used to approximate the broad patterns of global dissemination of bla $a_{\mathrm{NDM}}$. Our results 
252 are consistent with the spread stabilising between 2013-2015, with a gradual decline in diversity afterwards

253 (Supplementary Figure 16A). This observation supports a scenario whereby the global dissemination of NDM

254 took place over 8-10 years. Temporal diversity of bacterial genera was largely unchanged, consistent with bla $a_{\mathrm{NDM}}$

255 having been highly mobile across genera since at least 2005 (Supplementary Figure 16B).

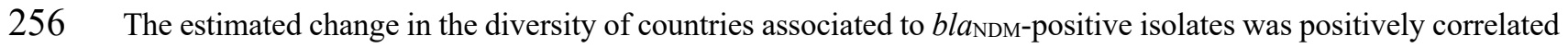

257 with other metadata categories (Supplementary Figure 17) suggesting it holds information which can be leveraged

258 to reconstruct dissemination trends. The strongest correlation was found between the diversity of countries and

259 plasmid backbones ( $\rho=0.864$ [0.691-0.964]) supporting a strong dependence between the two (Supplementary

260 Figure 17B). To further investigate this relationship, we assessed the correlation between genetic and geographic

261 distance between pairs of confirmed plasmid contigs (tested for IncF, IncX3, IncC, IncN2 and confirmed plasmid

262 contigs $>10 \mathrm{~kb}$ ) as a function of the distance downstream of bla $a_{\text {NDM }}$ gene (Supplementary Figure 18, see Methods).

263 No relationship was detected for IncX3 and IncN2 plasmids (Supplementary Figure 18A and B) likely due to the

264 lack of long plasmid sequences and deficient geographic distance between pairs of plasmids as both replicon types

265 are mostly localized to China and India respectively (Supplementary Figure 3). However, in all other cases aside

266 from IncN2 plasmids, a peak in the correlation recovered between genetic and geographical distance was observed

267 immediately downstream of bla

268 (Supplementary Figure 18). More importantly, in IncF and IncC, and other confirmed plasmid contigs, a notable

269 and gradual increase in the strength of correlation was noted further downstream as more plasmid sequence is

270 included in the analysis (Supplementary Figure 18B, C, and D). These trends suggest that plasmids carrying

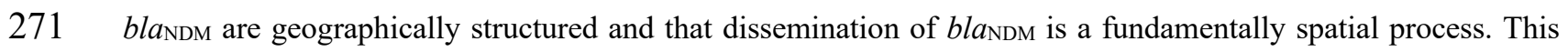

272 would be consistent with the existence of plasmid niches: settings to which particular plasmids are more adapted. 


\section{Discussion}

274 In this study, we have characterised the extant structural variation around bla $a_{\mathrm{NDM}}$ in a large global dataset in order

275 to reconstruct its evolutionary history and the main actors underlying its spread. Our results, largely summarized

276 in Figure 3, highlight an ancestral background of bla $a_{\mathrm{NDM}}$ as well as several insertion events and a myriad of other

277 genetic reshuffling, together pointing to an early emergence of bla $a_{\mathrm{NDM}}$ followed by a more recent and rapid

278 dissemination globally. Genetic reshuffling and mobilization of bla $a_{\mathrm{NDM}}$ by multiple transposons aided its rapid

279 dissemination via a multitude of plasmid backbones.

280 We go beyond previous smaller studies by dating the MRCA of the hypothesised ancestral form - the transposon

281 Tn125, together with bla $a_{\mathrm{NDM}}$ in its chimeric form ${ }^{10}$ - to pre-1990, and possibly well back into the mid- $20^{\text {th }}$ century.

282 A likely scenario is an origin in Acinetobacter in the Indian subcontinent. We note that $\mathrm{Tn} 125$ is mostly present

283 in Acinetobacter and Klebsiella species and it is likely this transposon played an important role in early plasmid

284 jumps of bla $a_{\mathrm{NDM}}$, given it is the dominant transposon in our comprehensive dataset which encompasses the

285 ancestral genetic background of bla $a_{\mathrm{NDM}}-$ groS/groL genes and ISCR27 sequence. We also estimate the formation

286 of a secondary transposon, involving Tn3000, which remobilized the region likely in Klebsiella species sometime

287 between the 1980s and early 2000s. However, we suggest Tn3000 likely played a lesser role in the early spread

288 of bla NDM $_{\text {as }}$ it does not include the ISCR 27 found at least partially preserved in many samples.

289 In total, 31 different putative transposons were identified within our dataset. Their role, together with integrons

290 and other transposable elements, is likely mostly minor or disruptive, as suggested for ISCR1. However, we do

291 identify IS26 as of interest, given it frequently forms putative transposons in our dataset, especially in IncF

292 plasmids. IS26 is known for its increased activity and rearrangement of plasmids in clinical isolates ${ }^{55}$ and has been

293 observed to drive within-plasmid heterogeneity even in a single E. coli isolate ${ }^{56}$. Thus, IS26 flanked pseudo-

294 composite transposons likely represent the most important contributor to genetic reshuffling of blandM in recent

295 times.

296 Our assessment of temporal diversity of countries of origin of bla $\mathrm{NDM}_{\mathrm{N}}$ positive isolates supports a globalisation

297 peak in 2013-2015. Such a rapid 8-10 year world-wide spread has been suggested for other important mobile

298 resistance genes such as the $m c r-1$ gene, mediating colistin resistance ${ }^{57}$. The extent to which this model of 'rapid

299 global spread' applies to other transposon-borne resistance elements remains to be determined.

300 We found 33 different plasmid types carrying bla $\mathrm{NDM}_{\mathrm{N}}$ and a positive correlation between genetic distance

301 calculated for differing lengths of plasmid backbones and geographic distances of sampling locations. Such an

302 observation is consistent with the existence of a constraint on plasmid spread, i.e. plasmid niches. Such niches

303 may exist as a result of local ecological and evolutionary pressures acting on particular plasmid backbones. Such

304 forces may include country boundaries limiting population movement, region-specific patterns in antibiotic usage,

305 influence of co-resistance, plasmid fitness costs, conjugation rates and copy numbers, the narrow host range of

306 the majority of bacterial plasmids ${ }^{58}$, or plasmids being associated with particular locations or environmental

307 niches ${ }^{59}$, all may contribute to restricting plasmid geographical range. Thus, an introduction of another plasmid

308 into a foreign plasmid niche may lead to plasmid loss or fast adaptation by, for instance, acquisition of resistance

309 and other accessory elements. This hypothetical scenario also provides an opportunity for resistance to spread by 
310 transposition or recombination, by which a new resistance gene could establish itself into another plasmid niche.

311 In the case of bla $a_{\mathrm{NDM}}$, this would also imply that after the initial introduction of bla $a_{\mathrm{NDM}}$ to a geographic region,

312 dissemination and persistence of the gene could proceed idiosyncratically - selection for carbapenem resistance

313 being just one of many selective pressures acting on plasmid diversity.

314 The importance of transposon movement has been previously demonstrated by work on plasmid networks ${ }^{58,60}$, as

315 well as several papers promoting a Russian-doll model of resistance mobility ${ }^{57,61}$. Considering our results, we

316 suggest a conceptual framework of AMR gene dissemination across genera where plasmid mobility is for the most

317 part restricted. Although plasmids can facilitate rapid spread within species and geographical regions, plasmid

318 transfer is not the main driver of widespread dissemination. Instead, most plasmid horizontal transfers are likely

319 only transient, with plasmids generally failing to establish themselves in the new bacterial host. Though, such

320 aborted plasmid exchanges still provide a crucial opportunity for between-plasmid transposon jumps and genetic

321 recombination to spread AMR genes across bacterial species. 


\section{Compiling the curated dataset of NDM sequences}

324 We compiled an extensive dataset of 6155 bacterial genomes carrying the bla available databases. 2632, 1158, and 1379 fully assembled genomes were downloaded from NCBI Reference

326 Sequence Database ${ }^{35,62}$ (RefSeq; accessed on $15^{\text {th }}$ of April 2021), NCBI's GenBank ${ }^{36}$ (accessed on $15^{\text {th }}$ of April 2021), and EnteroBase (accessed on $27^{\text {th }}$ of April 2021) ${ }^{37}$ respectively. The EnteroBase repository was screened for bla $\mathrm{NDM}_{\mathrm{M}}$ using BlastFrost (v1.0.0) ${ }^{63}$ allowing for one mismatch. In addition, we used the Bitsliced Genomic Signature Index (BIGSI) tool (v0.3) ${ }^{64}$ to identify all Sequence Read Archive (SRA) unassembled reads which

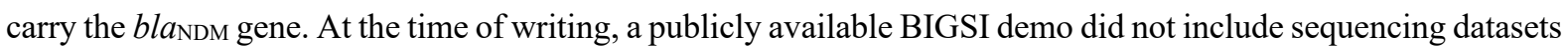
from after December 2016. Therefore, we manually indexed and screened an additional 355,375 SRA bacterial sequencing datasets starting from January 2017 to January 2019. We required the presence of 95\% of bla NDM- $1_{k-}$ mers to identify NDM-positive samples from raw SRA reads. This led to the inclusion of a further 882 isolates. The dataset also included 104 NDM-positive genomes from 79 hospitalized patients across China and 25 livestock farms selected from two previous studies ${ }^{65,66}$. These were sequenced using paired-end Illumina (Illumina HiSeq 2500) and PacBio (PacBio RS II). The sequencing reads are available on the Short Read Archive (SRA) under accession number PRJNA761884. All reads were de novo assembled using Unicycler (v0.4.8) ${ }^{67}$ with default parameters while also specifying hybrid mode for those isolates for which we had both Illumina short-read and PacBio long read sequencing data. Spades (v3.11.1) ${ }^{68}$ was applied, without additional polishing, for cases where Unicycler assemblies failed to resolve.

341 Assembled genomes were retained when they derived from a single BioSample identifier. Contigs carrying the

342 bla $a_{\mathrm{NDM}}$ gene were identified using BLAST (v2.10.1+) $)^{46}$. Forty-eight contigs were found to carry more than one copy of bla $a_{\mathrm{NDM}}$ and were not included in our analyses and eighty-eight contigs were excluded due to having partial $(<90 \%)$ bla $_{\mathrm{NDM}}$ hits. Fourteen assemblies had a single bla $a_{\mathrm{NDM}}$ gene split into two contigs; these 28 contigs were also excluded. Several contigs were also removed due to poor assembly quality. The filtering resulted in a dataset of 7148 contigs ( 6,155 samples) which were used in all subsequent analyses. Of these, 958 assembled genomes were found to contain bla NDM $_{\text {on }}$ multiple (mostly two) contigs, each harbouring a single and complete copy of bla $a_{\mathrm{NDM}}$. Even though the information about sequencing platform or assembly methods of most samples from RefSeq, GenBank and Enterobase databases could not be determined, the distribution of bla $a_{\mathrm{NDM}}$-positive contig lengths (Supplementary Figure 1) reveals they are likely to be based on short reads with the minority of contigs, mostly from RefSeq, reaching the quality of a hybrid de novo assembly. The full table of contigs and metadata considered is available as Supplementary Data 1.

355 Full metadata for each genome was collected from its respective database and the R package 'taxize ${ }^{969}$ used to

356 retrieve taxonomic information for each sample. In the case of samples for which exact sampling coordinates were 
357 not provided, Geocoding API from Google cloud computing services was used to retrieve coordinates based on

358 location names.

359 Coding sequences (CDS) of all NDM-positive contigs were annotated using the annotation tool Prokka (v1.14.6) ${ }^{70}$

360 and Roary (v3.13.0) ${ }^{71}$ run with minimum blastp percentage identity of $90 \%$ (-i 0.9 ) and disabled paralog splitting

361 (-s). To identify plasmid replicon types ${ }^{72}$, contigs were screened against the PlasmidFinder replicon database

362 (version 2020-02-25) ${ }^{44}$ using BLAST (v2.10.1+) ${ }^{46}$ where only BLAST hits with a minimum coverage of $80 \%$ and

363 percentage identity of $\geq 95 \%$ were retained. In cases where two or more replicon hits were found at overlapping

364 positions on a contig, the one with the higher percentage identity was retained. Identified plasmid types were used

365 to cluster contigs into broader plasmid groups: IncX3, IncF, IncC, IncN2, IncHI1B, IncHI2, and other (Figure

366 1D).

367 NDM-positive contigs were also screened against a dataset of complete bacterial plasmids. Bacterial plasmid 368 references were obtained from $\operatorname{RefSeq}^{35}$ and curated as described in Acman et al. ${ }^{73}$ Mash, a MinHash based 369 genome distance estimator ${ }^{74}$, was applied with default settings to evaluate pairwise genetic distances between 370 contig sequences and plasmid references. Contig-reference hits with less than 0.05 Mash distance and less than $37120 \%$ difference in length were retained. Additional pruning was implemented such that, for each contig analysed, 372 only the $10 \%$ of best scoring plasmid reference hits were retained. A table of pairwise genetic distances between 373 contigs and references was represented as a network which was then analysed with the infomap ${ }^{75}$ community 374 detection algorithm. Contigs were annotated according to their community membership and the network was 375 visualized using Cytoscape ${ }^{76}$ (Supplementary Figure 4).

377 Resolving structural variants of NDM-positive contigs

378 A novel alignment-based approach was used to identify stretches of homology (i.e., maximal alignable regions) 379 as well as structural variations across all contigs upstream and downstream of bla $a_{\mathrm{NDM}}$ gene. A conceptual 380 illustration of the method is presented in Figure 2. First, contigs carrying bla 381 bla $a_{\mathrm{NDM}}$ gene was located on the positive-sense DNA strand (i.e., facing 5' to 3' direction). A discontiguous Mega 382 BLAST (v2.10.1+) ${ }^{47}$ search with default settings was then applied against all pairs of retained contigs. This 383 method was selected over the regular Mega BLAST implementation as it is comparably fast, but more permissive 384 towards dissimilar sequences with frequent gaps and mismatches. BLAST hits including a complete bla 385 represent maximal stretches of homology around the gene for every pair of contigs. The analysis continues by 386 considering only portions of BLAST hits: (i) the start of bla $a_{\mathrm{NDM}}$ gene and the downstream sequence or (ii) the end 387 of the bla $a_{\mathrm{NDM}}$ gene and the upstream sequence depending on the analysis at hand: the downstream or the upstream 388 analysis respectively. This trimming of BLAST hits establishes bla $a_{\mathrm{NDM}}$ as an anchor and enables comparisons to 389 be made across all samples.

390 A table of BLAST hits can be considered as a network (graph), where each pair of contigs (i.e., nodes) are 391 connected by the edge weighted by the length of the BLAST hit. The algorithm proceeds with a stepwise network 392 analysis of BLAST hits. For this purpose, a 'splitting threshold' was introduced. Starting from zero which 393 represents the start/end position of bla $a_{\mathrm{NDM}}$ gene, the threshold is gradually increased by $10 \mathrm{bp}$. At each step, 
394 BLAST hits with a length lower than the value given by the 'splitting threshold' are excluded. Thus, as the 'splitting threshold' increases, a network of BLAST hits is also pruned and broken down into components - groups of interconnected nodes (contigs). It is expected that contigs within each component share a homologous region downstream (or upstream) of bla $a_{\text {NDM }}$ at least of the length given by the threshold. It is therefore not possible for a single contig to be assigned to multiple components. Components of size $<10$ are labelled as 'Other Structural Variants'. Also, contigs that are shorter than the defined 'splitting threshold' and share no edge with any other contig are considered as 'cutting short'.

401 By tracking the splitting of the network as the 'splitting threshold' is increased, one can determine clusters of 402 homologous contigs at any given position downstream or upstream from the anchor gene (here bla $a_{\mathrm{NDM}}$ ), as well 403 as the homology breakpoint. The precision of the algorithm is directly influenced by the step size, in this case 10 404 bp, and the alignment algorithm, in this case discontiguous Mega BLAST. We assessed the precision of the algorithm on the tree of structural variations downstream of bla $a_{\mathrm{NDM}}$ (Figure 3). To this end, we compared extended $50 \mathrm{bp}$ sequence fragments of each branching point in the tree checking for missed homologies and comparing Mash distances between pairs of branched-out contigs. We found no similarities among 50bp fragments of any split branches. The described algorithm is available at https://github.com/macman123/track_structural_variants.

\section{Analysing the contig overhanging reads}

411 To investigate the reasons behind a number of distinctively short blandM-carrying contigs, we mapped 781 raw 412 Illumina paired-end sequencing reads (originally downloaded from SRA) back to their matching contigs. The 413 mapping was done using BBMap ${ }^{77}$ (v38.59; maxindel $=0$ and minratio $=0.2$ settings). Within the output SAM 414 file, only the overhanging reads with the CIGAR string matching the "[0-9]* $M[0-9] * S$ " regular expression were selected. All overhangs of reads $\geq 50 \mathrm{bp}$ were screened against ISFinder database ${ }^{49}$.

\section{Molecular tip-dating analysis.}

418 The 112 complete Tn 125 and 73 complete Tn3000 contigs with a known collection date and harbouring bla $a_{\mathrm{NDM}}$ 419 were sequentially aligned (--pileup flag) using Clustal Omega (v1.2.3) ${ }^{78}$ specifying the bla $a_{\mathrm{NDM}-1}$ sequence 420 (FN396876.1) as a profile. Each alignment was manually inspected using UGENE (v38.0) ${ }^{79}$. The ancestral (i.e., 421 root) sequence was determined by evaluating SNP frequencies over time (Supplementary Figure 9 and 10). Due 422 to a short sampling time span and relatively few mutations present, it is unlikely that any one non-ancestral SNP 423 has become dominant in the population. Therefore, we expect the ancestral sequence to have a higher SNP 424 frequency in earlier years.

425 We find that, in all but two cases, the consensus sequence of an alignment displaying this behaviour. The first 426 exception is the consensus sequence allele of $\operatorname{Tn} 125$ at the variable position 441 (Supplementary Figure 9). This 427 allele has a low frequency in 2009. However, by inspecting the allele frequency table, we observe the low 428 frequency is based on a single sample. Leaving out this early sample restores the desired frequency pattern; hence 
the consensus allele is considered ancestral in this case. The second exception is the variable position 449 in the case of the Tn3000 alignment (Supplementary Figure 10). The consensus allele ' $a$ ' is not found in the early sample from 2009. Both allele ' $t$ ', present in the early sample, and allele ' $a$ ' were found equally frequent in more recent samples. Thus, due to lack of other evidence, allele ' $t$ ' was considered ancestral. Determined ancestral sequences were used to evaluate temporal signal in the alignment, and in the subsequent rooting of phylogenetic trees.

434 Date randomization (10,000 iterations) and linear regression analyses were employed to estimate the presence of 435 temporal signal in the alignment ${ }^{80-82}$ (Supplementary Figures 11 and 12). Tn 125 and Tn3000 showed significant 436 temporal signal using simple regression ( $p=0.0356$ and $p=0.0456$ respectively) and date randomization (true 437 evolutionary rate quantiles $>0.95$ ).

438 Bayesian based molecular dating approaches were implemented in BEAST2 (v2.6.0) ${ }^{83}$ and BactDating $^{84}$ to infer 439 the date of the emergence of the two transposons. Both BEAST2 and BactDating were run specifying a strict prior 440 on the molecular clock. For BEAST2, the generalised time reversible (GTR) substitution model prior was used 441 together with three population size models: Coalescent Constant population, Coalescent Exponential population, 442 and Coalescent Bayesian Skyline. In addition, all BEAST2 and BactDating runs were supplied with a maximum 443 likelihood (ML) phylogenetic tree (starting tree prior) constructed from both transposon alignments using RAxML 444 (v8.2.12) $)^{85}$ with specified GTRCAT substitution model and rooted using the inferred ancestral sequences. The 445 chosen MCMC chain lengths for BactDating and BEAST2 runs were $10^{7}$ and $1.5 \times 10^{9}$ respectively to ensure 446 convergence. We evaluated effective sample sizes (ESS) of the posterior distributions using effectiveSize function 447 implemented in $\operatorname{cod} a^{86} \mathrm{R}$ package after discarding the first $20 \%$ of burn-in (Supplementary Figures 13 and 14). 448 All BEAST2 and BactDating runs successfully converged with ESS of the posteriors close to or above 200. 449 BEAST2 input files are available as xml files in Supplementary Data 3.

\section{Estimating Shannon entropy among NDM-positive contigs}

451 We estimated Shannon entropy ('diversity') for several categorizations of bla sampling, bacterial host genera, plasmid backbones (determined by mapping to plasmid reference sequences), and

453 ISs flanking the bla $a_{\mathrm{NDM}}$ gene. To estimate entropy of the population and to provide confidence intervals around 454 our estimates, we use bootstrapping with replacement (1000 iterations). At each iteration, entropy was estimated for a sampled set of contigs $(X)$ classified into $n$ unique categories according to the following formula:

$$
H(X)=-\sum_{i=1}^{n} P\left(x_{i}\right) \log P\left(x_{i}\right)
$$
abundance of equally likely categories, while lower entropy indicates a limited number of categories. 
bioRxiv preprint doi: https://doi.org/10.1101/2021.01.14.426698; this version posted September 27, 2021. The copyright holder for this preprint (which was not certified by peer review) is the author/funder, who has granted bioRxiv a license to display the preprint in perpetuity. It is made available under aCC-BY-NC 4.0 International license.

\section{Estimating correlation between genetic and geographic distance}

462 Geographic distance between pairs of samples was determined using their sampling coordinates and the geodist ${ }^{87}$

463 R package. Exact Jaccard distance (JD) was used as a measure of the genetic distance calculated using the tool

464 Bindash (v0.2.1) ${ }^{88}$ with $k$-mer size equal to $21 \mathrm{bp}$. The JD is defined as the fraction of total $k$-mers not shared

465 between two contigs. JD between all pairs of contigs was first calculated on a 1000bp stretch of DNA downstream

466 of bla $a_{\mathrm{NDM}}$ start codon continuing with a 500bp increments. At each increment, the two distance matrices (genetic

467 and geographic) were assessed using the mantel function (Spearman correlation and 99 permutations) from the

$468 v \operatorname{van}^{89}$ package in $\mathrm{R}$. The correlation between genetic and geographic distance, was plotted as a function of

469 distance from bla NDM gene (Supplementary Figure 17). 


\section{Acknowledgements}

471 M.A. was supported by a Ph.D. scholarship from University College London. H.W. is supported by National

472 Natural Science Foundation of China (81625014). L.v.D., H.W. and F.B. acknowledge financial support from the

473 Newton Fund UK-China NSFC initiative (MRC Grant MR/P007597/1 and 81661138006). L.v.D. and F.B. are

474 supported from a Wellcome Institutional Strategic Support Fund (ISSF3) - AI in Healthcare (19RX03). F.B.

475 additionally acknowledges support from the BBSRC GCRF scheme and the National Institute for Health Research

476 University College London Hospitals Biomedical Research Centre. L.v.D is supported by a UCL Excellence

477 Fellowship. M.A., L.v.D and F.B. acknowledge UCL Biosciences Big Data equipment grant from BBSRC

478 (BB/R01356X/1). L.P.S. is a Sir Henry Wellcome Postdoctoral Fellow funded by Wellcome (Grant

$479220422 / \mathrm{Z} / 20 / \mathrm{Z}$ ). The funders had no role in study design, data collection, interpretation of results, or the decision

480 to submit the work for publication. Lastly, M.A. would like to thank Nicola de Maio for informal discussions

481 which led to the idea for the algorithm used to track structural variants.

\section{Contributions}

483 M.A., F.B., L.v.D. and H.W. conceived the project and designed the experiments. M.A., L.v.D., L.P.S., and N.L. 484 collected data from online repositories. R.W., Y.Y., Q.W., S.S, and H.C sequenced samples from Chinese 485 hospitals. M.A., L.v.D, and R.W. de novo assembled all the genomes. M.A. performed all the analyses under the 486 guidance of L.v.D and F.B. M.A., L.v.D. and F.B. take responsibility for the accuracy and availability of the 487 results. M.A. wrote the paper with contributions from L.P.S., L.v.D., and F.B. All authors read and commented 488 on successive drafts and all approved the content of the final version.

\section{Competing interests}

490 The authors declare no financial or non-financial competing interests.

\section{Data availability}

492 The accession numbers of bacterial genomes obtained from the RefSeq, Enterobase and SRA databases are given 493 in the Supplementary Data 1. One hundred and four paired-end Illumina and PacBio sequencing data from China 494 are available on SRA under the BioProject accession number PRJNA761884. Whole genome de novo assemblies 495 are available on GenBank under the same BioProject accession number. Filtered dataset of 7148 bla $a_{\mathrm{NDM}}$ bearing 496 contigs is available on Figshare: 10.5522/04/16594784

\section{Code availability}

498 All software used in this research are listed in Methods. An implementation of the algorithm used to track structural variations around bla $a_{\mathrm{NDM}}$ is available at https://github.com/macman123/track structural variants. 
500

501

502

503

504

505

506

507

508

509

510

511

512

513

514

515

516

517

518

519

520

521

522

523

524

525

526

527

528

529

530

531

532

533

534

535

536

537

538

539

\section{References}

1. $\mathrm{Wu}, \mathrm{W}$. et al. NDM metallo- $\beta$-lactamases and their bacterial producers in health care settings. Clinical Microbiology Reviews vol. 32 (2019).

2. Yong, D. et al. Characterization of a new metallo- $\beta$-lactamase gene, bla NDM-1, and a novel erythromycin esterase gene carried on a unique genetic structure in Klebsiella pneumoniae sequence type 14 from India. Antimicrob. Agents Chemother. 53, 5046-5054 (2009).

3. Struelens, M. J. et al. New Delhi metallo-beta-lactamase 1-producing Enterobacteriaceae: emergence and response in Europe. Eurosurveillance 15, 19716 (2010).

4. Kumarasamy, K. K. et al. Emergence of a new antibiotic resistance mechanism in India, Pakistan, and the UK: A molecular, biological, and epidemiological study. Lancet Infect. Dis. 10, 597-602 (2010).

5. Poirel, L., Dortet, L., Bernabeu, S. \& Nordmann, P. Genetic Features of bla NDM-1-Positive

6. Castanheira, M. et al. Early dissemination of NDM-1- and OXA-181-producing Enterobacteriaceae in Indian hospitals: Report from the SENTRY Antimicrobial Surveillance Program, 2006-2007.

Antimicrob. Agents Chemother. 55, 1274-1278 (2011).

7. Jones, L. S. et al. Plasmid carriage of blaNDM-1in clinical Acinetobacter baumannii isolates from India.

8. Roca, I. et al. Molecular characterization of NDM-1-producing Acinetobacter pittii isolated from Turkey in 2006. J. Antimicrob. Chemother. 69, 3437-3438 (2014).

9. Poirel, L., Bonnin, R. A. \& Nordmann, P. Analysis of the resistome of a multidrug-resistant NDM-1producing Escherichia coli strain by high-throughput genome sequencing. Antimicrob. Agents Chemother. 55, 4224-4229 (2011).

10. Toleman, M. A., Spencer, J., Jones, L. \& Walsha, T. R. bla NDM-1 is a chimera likely constructed in Acinetobacter baumannii. Antimicrob. Agents Chemother. 56, 2773-2776 (2012).

11. Partridge, S. R. \& Iredell, J. R. Genetic Contexts of bla NDM-1. Antimicrobial Agents and Chemotherapy vol. 56 6065-6067 (2012).

12. Partridge, S. R. \& Iredell, J. R. Genetic Contexts of bla NDM-1. Antimicrobial Agents and Chemotherapy vol. 56 6065-6067 (2012).

13. Toleman, M. A., Bennett, P. M. \& Walsh, T. R. ISCR Elements: Novel Gene-Capturing Systems of the 21st Century? Microbiol. Mol. Biol. Rev. 70, 296-316 (2006).

14. Ilyina, T. S. Mobile ISCR elements: Structure, functions, and role in emergence, increase, and spread of blocks of bacterial multiple antibiotic resistance genes. Molecular Genetics, Microbiology and Virology vol. 27 135-146 (2012).

15. Poirel, L. et al. Tn125-related acquisition of blaNDM-like genes in Acinetobacter baumannii. Antimicrob. Agents Chemother. 56, 1087-1089 (2012).

16. Sekizuka, T. et al. Complete Sequencing of the blaNDM-1-Positive IncA/C Plasmid from Escherichia coli ST38 Isolate Suggests a Possible Origin from Plant Pathogens. PLoS One 6, e25334 (2011).

17. McArthur, A. G. et al. The comprehensive antibiotic resistance database. Antimicrob. Agents Chemother. 57, 3348-3357 (2013). 
540 18. Basu, S. Variants of the New Delhi metallo- $\beta$-lactamase: New kids on the block. Future Microbiology vol. 15 465-467 (2020).

19. Baraniak, A. et al. NDM-producing Enterobacteriaceae in Poland, 2012-14: inter-regional outbreak of Klebsiella pneumoniae ST11 and sporadic cases. J. Antimicrob. Chemother. 71, 85-91 (2016).

20. Rahman, M. et al. Prevalence and Molecular Characterization of New Delhi Metallo-Beta-Lactamases in Multidrug-Resistant Pseudomonas aeruginosa and Acinetobacter baumannii from India. Microb. Drug Resist. 24, 792-798 (2018).

21. Hu, H. et al. Novel plasmid and its variant harboring both a bla(NDM-1) gene and type IV secretion system in clinical isolates of Acinetobacter lwoffii. Antimicrob. Agents Chemother. 56, 1698-702 (2012).

22. Yang, Q. et al. Dissemination of NDM-1-producing Enterobacteriaceae mediated by the IncX3-type plasmid. PLoS One 10, (2015).

23. Wailan, A. M. et al. Genetic contexts of blaNDM-1 in patients carrying multiple NDM-producing strains. Antimicrob. Agents Chemother. 59, 7405-7410 (2015).

24. Rasheed, J. K. et al. New Delhi Metallo- $\beta$-Lactamase-producing Enterobacteriaceae, United States. Emerg. Infect. Dis. 19, 870 (2013).

25. Poirel, L. et al. Tn125-related acquisition of blaNDM-like genes in Acinetobacter baumannii.

(2012)

26. Campos, J. C. et al. Characterization of Tn3000, a Transposon Responsible for blaNDM-1 Dissemination among Enterobacteriaceae in Brazil, Nepal, Morocco, and India. Antimicrob. Agents Chemother. 59, 7387-95 (2015).

27. Feng, Y., Liu, L., McNally, A. \& Zong, Z. Coexistence of two blaNDM-5 genes on an IncF plasmid as

28. Zhao, Q.-Y. et al. IS 26 Is Responsible for the Evolution and Transmission of bla NDM -Harboring Plasmids in Escherichia coli of Poultry Origin in China . mSystems (2021) doi:10.1128/MSYSTEMS.00646-21.

29. Lynch, T. et al. Molecular evolution of a klebsiella pneumoniae st278 isolate harboring blandm-7 and

30. Huang, T. W. et al. Copy Number Change of the NDM-1 Sequence in a Multidrug-Resistant Klebsiella

31. Datta, S. et al. Spread and exchange of bla NDM-1 in hospitalized neonates: role of mobilizable genetic elements. Eur. J. Clin. Microbiol. Infect. Dis. 36, 255-265 (2017). carbapenemase gene. Antimicrob. Agents Chemother. 60, 3032-3040 (2016). lactamase. Nat. Chem. Biol. 12, 516-522 (2016). via outer membrane vesicles. J. Antimicrob. Chemother. 72, 2201-2207 (2017).

578 35. O'Leary, N. A. et al. Reference sequence (RefSeq) database at NCBI: current status, taxonomic expansion, and functional annotation. Nucleic Acids Res. 44, D733-45 (2016). 
36. Benson, D. A. et al. GenBank. Nucleic Acids Res. 41, (2013).

37. Zhou, Z., Alikhan, N. F., Mohamed, K., Fan, Y. \& Achtman, M. The EnteroBase user's guide, with case studies on Salmonella transmissions, Yersinia pestis phylogeny, and Escherichia core genomic diversity. Genome Res. 30, 138-152 (2020).

38. Souvorov, A., Agarwala, R. \& Lipman, D. J. SKESA: Strategic k-mer extension for scrupulous

39. Tatusova, T., Ciufo, S., Fedorov, B., O’Neill, K. \& Tolstoy, I. RefSeq microbial genomes database: New representation and annotation strategy. Nucleic Acids Res. 42, D553-D559 (2014).

40. Chavda, K. D. et al. Comprehensive genome analysis of carbapenemase-producing Enterobacter spp.: New insights into phylogeny, population structure, and resistance mechanisms. MBio 7, (2016).

41. Ashton, P. M. et al. Identification of Salmonella for public health surveillance using whole genome sequencing. PeerJ 2016, e1752 (2016).

42. Sahl, J. W. et al. Phylogenetic and genomic diversity in isolates from the globally distributed Acinetobacter baumannii ST25 lineage. Sci. Rep. 5, (2015).

43. Bonnin, R. A. et al. Dissemination of New Delhi metallo- $\beta$-lactamase-1-producing Acinetobacter baumannii in Europe. Clin. Microbiol. Infect. 18, E362-E365 (2012).

44. Carattoli, A. et al. In Silico Detection and Typing of Plasmids using PlasmidFinder and Plasmid

45. Roach, D. et al. Whole Genome Sequencing of Peruvian Klebsiella pneumoniae Identifies Novel Plasmid Vectors Bearing Carbapenem Resistance Gene NDM-1. Open Forum Infect. Dis. 7, (2020). Camacho, C. et al. BLAST+: architecture and applications. BMC Bioinformatics 10, 421 (2009). 18, 440-445 (2002).

48. Schnetz, K. \& Rak, B. IS5: A mobile enhancer of transcription in Escherichia coli. Proc. Natl. Acad. Sci. U. S. A. 89, 1244-1248 (1992).

49. Siguier, P., Perochon, J., Lestrade, L., Mahillon, J. \& Chandler, M. ISfinder: the reference centre for bacterial insertion sequences. Nucleic Acids Res. 34, D32-D36 (2006).

50. Harmer, C. J., Pong, C. H. \& Hall, R. M. Structures bounded by directly-oriented members of the IS26 occurs via a translocatable unit that includes a single IS26 and preferentially inserts adjacent to another IS26. MBio 5, (2014).

613

52. Li, J. et al. Sequential Isolation in a Patient of Raoultella planticola and Escherichia coli Bearing a Novel ISCR1 Element Carrying blaNDM-1. PLoS One 9, e89893 (2014).

19, 23-40 (2018).

616 54. Harmer, C. J. \& Hall, R. M. An analysis of the IS6/IS26 family of insertion sequences: Is it a single 617 family? Microb. Genomics 5, (2019).

618 55. He, S. et al. Insertion sequence IS26 reorganizes plasmids in clinically isolated multidrug-resistant bacteria by replicative transposition. MBio 6, 1-14 (2015). 
56. He, D. D. et al. Antimicrobial resistance-encoding plasmid clusters with heterogeneous MDR regions driven by IS26 in a single Escherichia coli isolate. J. Antimicrob. Chemother. 74, 1511-1516 (2019).

57. Wang, R. et al. The global distribution and spread of the mobilized colistin resistance gene mcr-1. Nat. Commun. 9, 1-9 (2018).

58. Acman, M., van Dorp, L., Santini, J. M. \& Balloux, F. Large-scale network analysis captures biological

59. Shaw, L. et al. Niche and local geography shape the pangenome of wastewater- and livestock-associated

60. Redondo-Salvo, S. et al. Pathways for horizontal gene transfer in bacteria revealed by a global map of their plasmids. Nat. Commun. 2020111 11, 1-13 (2020).

61. Sheppard, A. E. et al. Nested Russian Doll-Like Genetic Mobility Drives Rapid Dissemination of the Carbapenem Resistance Gene blaKPC. Antimicrob. Agents Chemother. 60, 3767-3778 (2016).

62. Pruitt, K. D., Tatusova, T. \& Maglott, D. R. NCBI reference sequences (RefSeq): A curated nonredundant sequence database of genomes, transcripts and proteins. Nucleic Acids Res. 35, D61-D65 (2007).

63. Luhmann, N., Holley, G. \& Achtman, M. BlastFrost: Fast querying of 100,000s of bacterial genomes in Bifrost graphs. bioRxiv 1-24 (2020) doi:10.1101/2020.01.21.914168.

64. Bradley, P., den Bakker, H. C., Rocha, E. P. C., McVean, G. \& Iqbal, Z. Ultrafast search of all deposited bacterial and viral genomic data. Nat. Biotechnol. 37, 152-159 (2019).

65. Wang, R. et al. The prevalence of colistin resistance in Escherichia coli and Klebsiella pneumoniae isolated from food animals in China: coexistence of mcr-1 and blaNDM with low fitness cost. Int. J.

67. Wick, R. R., Judd, L. M., Gorrie, C. L. \& Holt, K. E. Unicycler: Resolving bacterial genome assemblies from short and long sequencing reads. PLoS Comput. Biol. 13, 1-22 (2017).

68. Bankevich, A. et al. SPAdes: a new genome assembly algorithm and its applications to single-cell sequencing. J. Comput. Biol. 19, 455-77 (2012). (2013).

70. Seemann, T. Prokka: rapid prokaryotic genome annotation. Bioinformatics 30, 2068-2069 (2014).

652 71. Page, A. J. et al. Roary: rapid large-scale prokaryote pan genome analysis. Bioinformatics 31, 36913693 (2015).

72. Orlek, A. et al. Plasmid classification in an era of whole-genome sequencing: Application in studies of antibiotic resistance epidemiology. Frontiers in Microbiology vol. 8 1-10 (2017).

73. Acman, M., van Dorp, L., Santini, J. M. \& Balloux, F. Large-scale network analysis captures biological features of bacterial plasmids. Nat. Commun. 11, 1-11 (2020). 
$66075 . \quad$ Rosvall, M. \& Bergstrom, C. T. Maps of random walks on complex networks reveal community structure. Proc. Natl. Acad. Sci. U. S. A. 105, 1118-1123 (2008).

76. Shannon, P. et al. Cytoscape: A software Environment for integrated models of biomolecular interaction networks. Genome Res. 13, 2498-2504 (2003).

664 77. Bushnell, B. BBMap: A Fast, Accurate, Splice-Aware Aligner. https://www.osti.gov/biblio/1241166 (2014).

666 78. Sievers, F. et al. Fast, scalable generation of high-quality protein multiple sequence alignments using Clustal Omega. Mol. Syst. Biol. 7, 539 (2011).

668 79. Okonechnikov, K., Golosova, O., Fursov, M. \& team, the U. Unipro UGENE: a unified bioinformatics toolkit. Bioinformatics 28, 1166-1167 (2012).

670

80. Rieux, A. \& Balloux, F. Inferences from tip-calibrated phylogenies: A review and a practical guide. Mol. Ecol. 25, 1911-1924 (2016).

81. Rambaut, A., Lam, T. T., Carvalho, L. M. \& Pybus, O. G. Exploring the temporal structure of heterochronous sequences using TempEst (formerly Path-O-Gen). Virus Evol. 2, 1-7 (2016).

82. Duchene, S. et al. Bayesian Evaluation of Temporal Signal In Measurably Evolving Populations. bioRxiv (2019) doi:10.1101/810697.

83. Bouckaert, R. et al. BEAST 2.5: An advanced software platform for Bayesian evolutionary analysis. PLoS Comput. Biol. 15, e1006650 (2019).

84. Didelot, X., Croucher, N. J., Bentley, S. D., Harris, S. R. \& Wilson, D. J. Bayesian inference of ancestral dates on bacterial phylogenetic trees. Nucleic Acids Res. 46, 1-11 (2018).

680

85. Stamatakis, A. RAxML version 8: A tool for phylogenetic analysis and post-analysis of large phylogenies. Bioinformatics 30, 1312-1313 (2014).

86. Plummer, M., Best, N., Cowles, K. \& Vines, K. CODA: convergence diagnosis and output analysis for MCMC - Open Research Online. R News 6, 7-11 (2006).

684 87. Padgham, M. \& Sumner, M. D. geodist: Fast, Dependency-Free Geodesic Distance Calculations. (2020).

686 88. Zhao, X. BinDash, software for fast genome distance estimation on a typical personal laptop. Bioinformatics 35, 671-673 (2019).

688 89. Oksanen, J. et al. vegan: Community Ecology Package. (2019). 


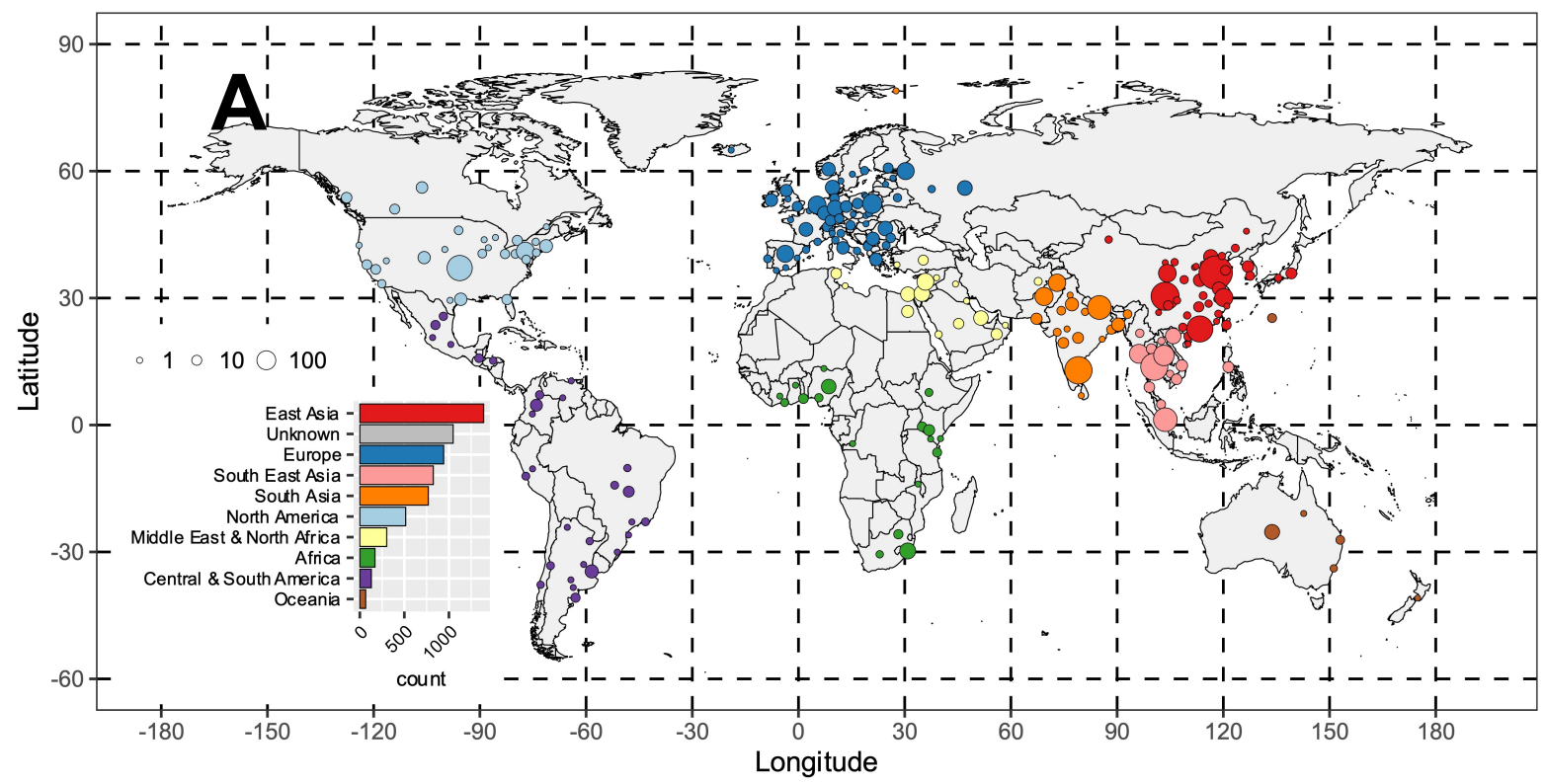

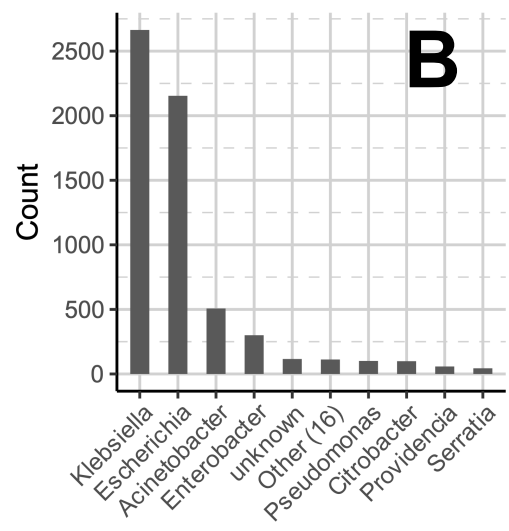

Genus

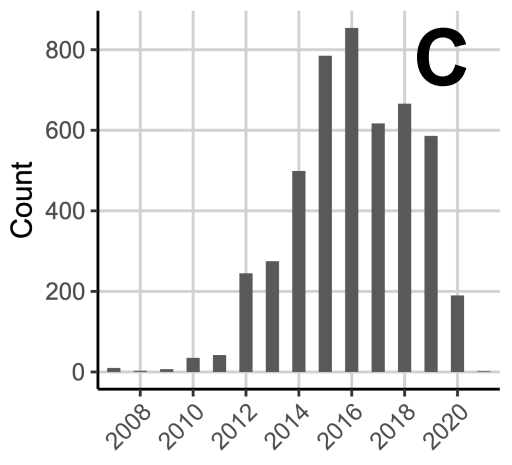

Collection Year

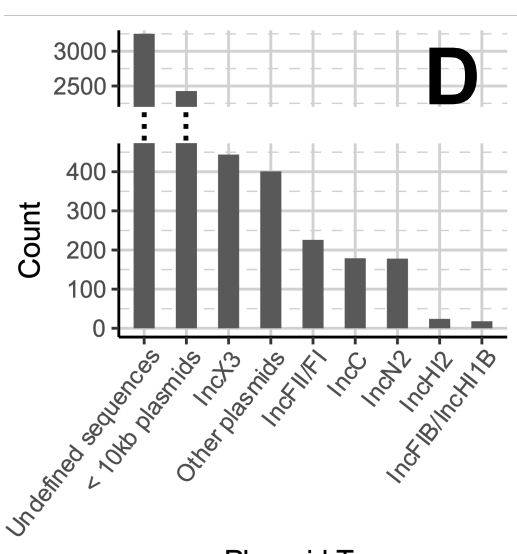

Plasmid Type
691

692 Figure 1. Composition of the global dataset of 6,155 NDM-positive samples. (A) Geographic

693 distribution of blanDM-positive assemblies. Points are coloured by geographic region and their size 694

695

696 reflects the number of samples they encompass. (B) Distribution of host bacterial genera of NDMpositive samples. (C) Distribution of sample collection years. (D) Distribution of contigs according to the plasmid backbone. 


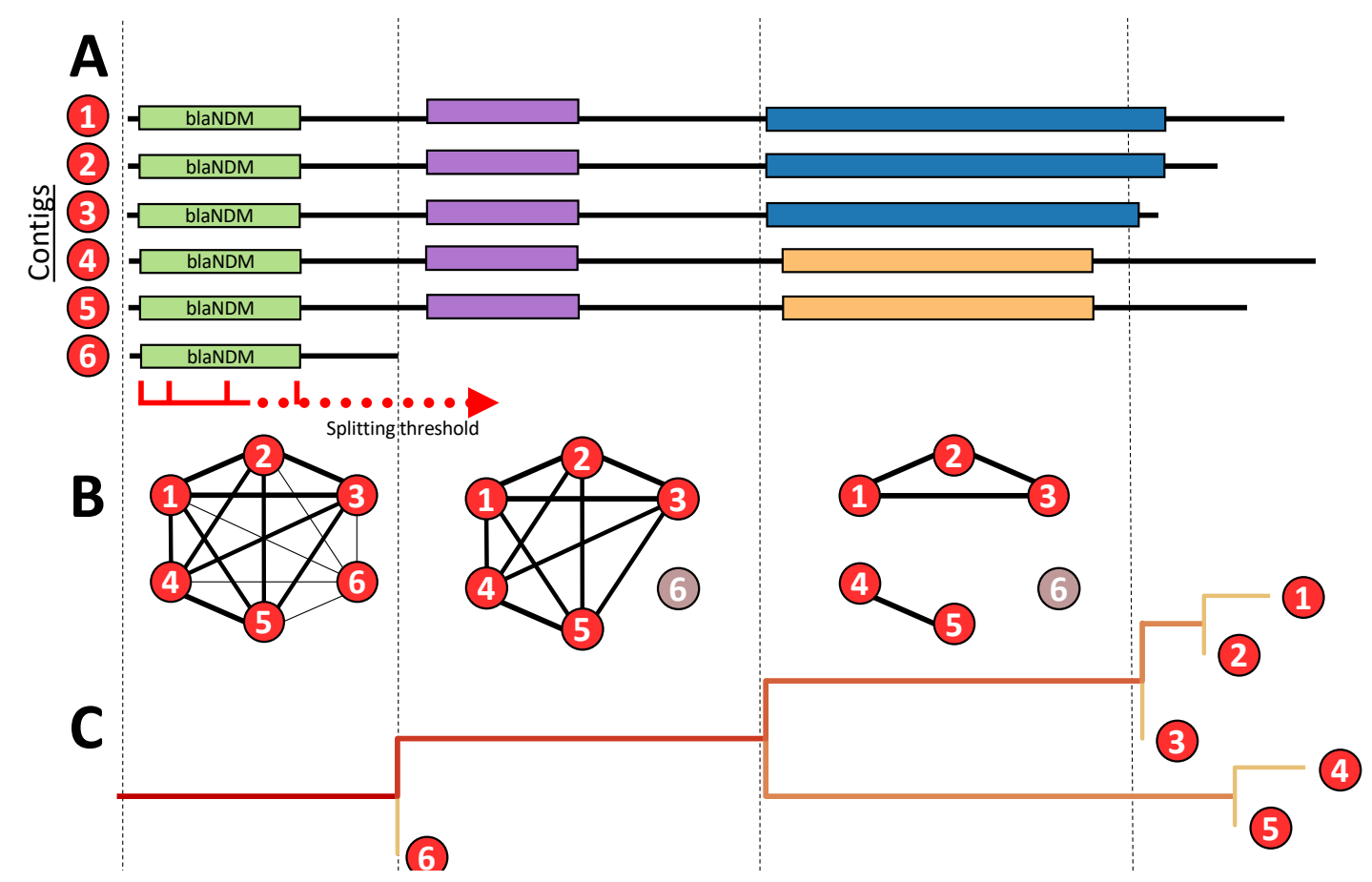

Figure 2. Schematic representation of the tracking algorithm splitting structural variants upstream or downstream of blandM gene. (A) A pairwise BLAST search is performed on all NDMpositive contigs. Starting from blaNDM and continuing downstream or upstream, the inspected region is gradually increased using the 'splitting threshold'. (B)

(B) At each step, a graph is constructed connecting contigs (nodes) that share a BLAST hit with a minimum length as given by the 'splitting threshold'. Contigs which have the same structural variant at the certain position of the threshold belong to the same graph component, while the short contigs are singled out. (C) The splitting is visualized as a tree where branch lengths are scaled to match the position within the sequence, and the thickness and the colour intensity of the branches correspond to the number of sequences carrying the homology. For more detailed explanation of the algorithm please refer to the Methods section. 


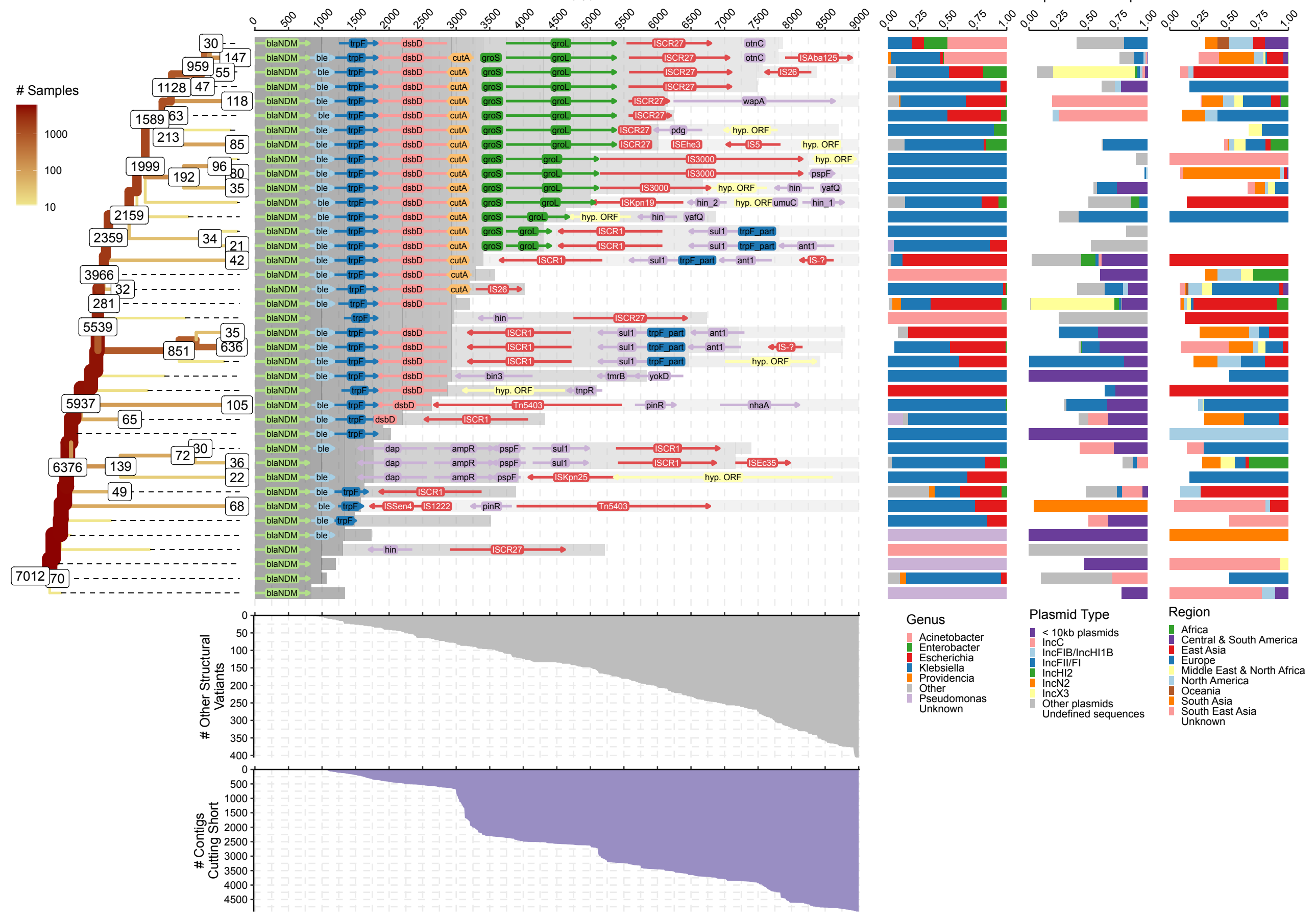


710 Figure 3. Splitting of structural variants downstream of blanDM. The 'splitting' tree for the most

711 common (i.e., $\geq 10$ contigs) structural variants is shown on the left-hand side. The labels on the nodes

712 indicate the number of contigs remaining on each branch. Labels of (yellow) branches with $<20$

713 contigs are not shown. The other contigs either belong to other structural variants or were removed

714 due to being too short in length. The number of contigs cutting short is indicated by the area chart at

715 the bottom. Similarly, the number of less common structural variants is indicated by the upper area

716 chart. Genome annotations provided by the Prokka and Roary pipelines of the most common

717 structural variants are shown in the middle of the figure. The homologous regions among structural

718 variants are indicated by the grey shading. Some of the structural variants and branches were

719 intentionally cut short even though their contigs were of sufficient size or longer. This was done to

720 prevent excessive bifurcation and to make the tree easier to interpret. In particular, branches with

721 more than $75 \%$ of contigs lost due to variation and short length were truncated. The distribution of

722 genera, plasmid backbones and geographical regions of samples that belong to a each of the

723 common structural variant is shown on the right-hand side. 

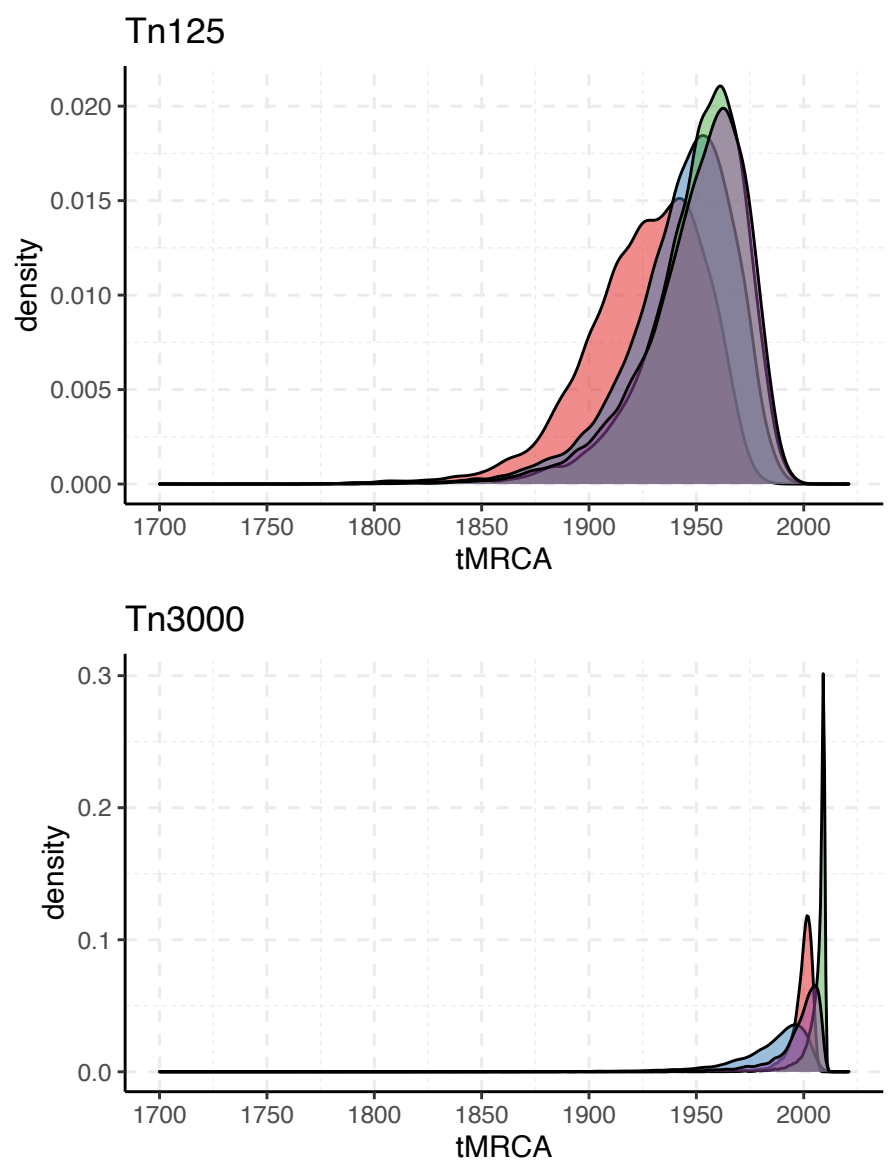

Molecular Dating Method

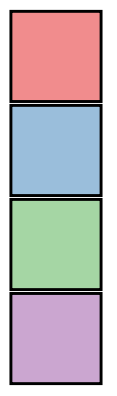

BactDating

tMRCA = $1925.62[1873.38-1971.86]$

BEAST2 - Coalescent Constant Population tMRCA = 1942.55 [1891-1983.98]

BEAST2 - Coalescent Exponential Population tMRCA $=1949.43$ [1901.82-1987.71]

BEAST2 - Coalescent Bayesian Skyline tMRCA = 1949.62 [1899.73-1988.67]

Molecular Dating Method

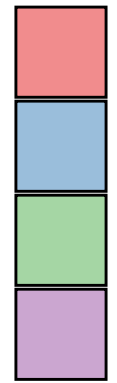

BactDating

tMRCA = 1998.63 [1986.65-2006.32]

BEAST2 - Coalescent Constant Population tMRCA = 1983.94 [1945.78-2007.71]

BEAST2 - Coalescent Exponential Population tMRCA = 2006.15 [1996.03-2009.5]

BEAST2 - Coalescent Bayesian Skyline tMRCA = 1996.11 [1966.85-2009.49]

Figure 4. Posterior density distributions of ancestral sequence age (i.e., root height) for the

$\operatorname{Tn} 125$ (A) and Tn3000 (B) transposons. The ancestral sequence emergence was estimated using two Bayesian tip-dating approaches implemented in BactDating and BEAST2. Three different population growth priors were used in case of BEAST2: Coalescent Constant Population, Coalescent Exponential Population, and Coalescent Bayesian Skyline as given by the colour scheme and legend at right. Median estimates with 95\% highest density interval (HDI) are provided in the panel legends. 


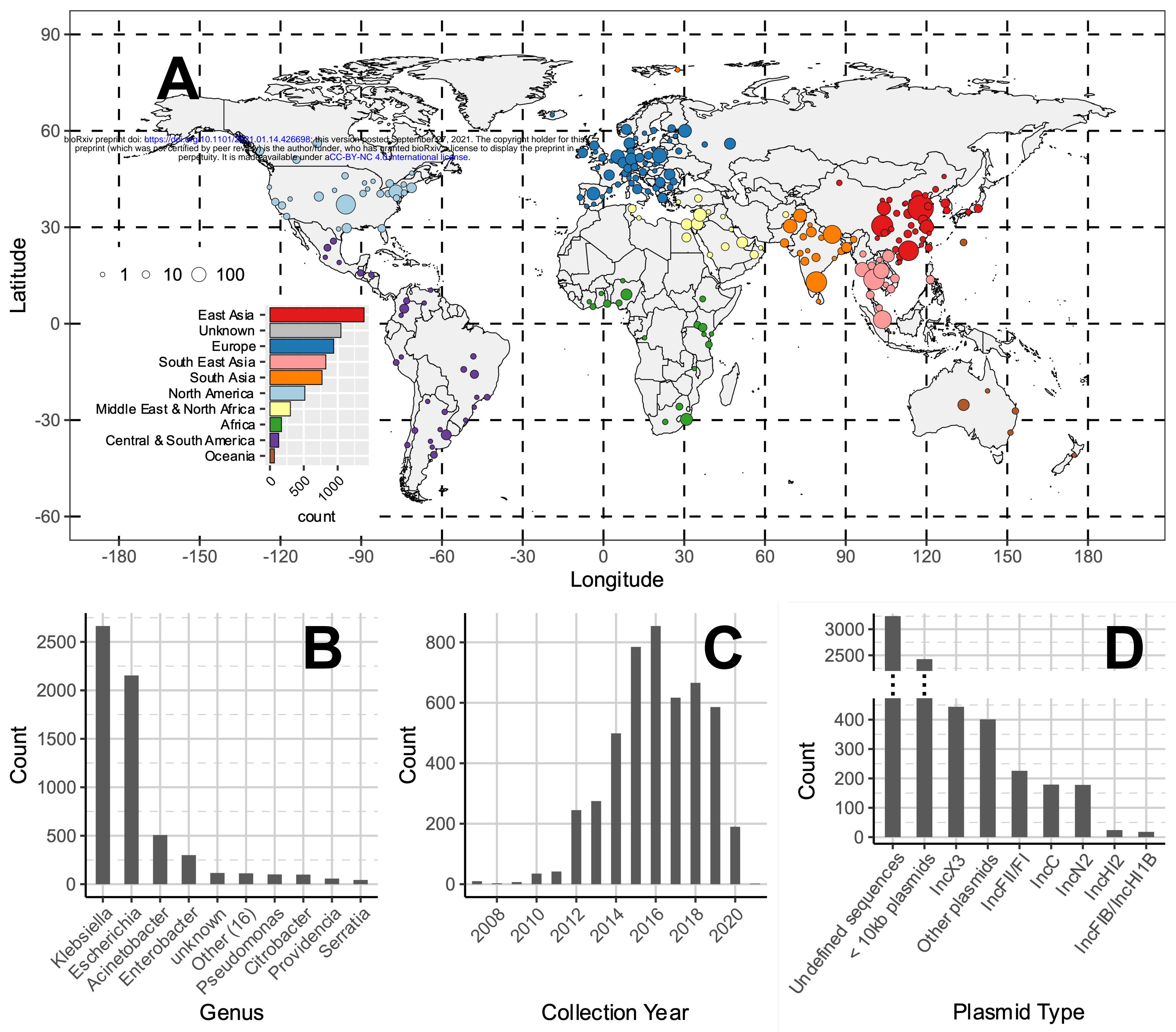




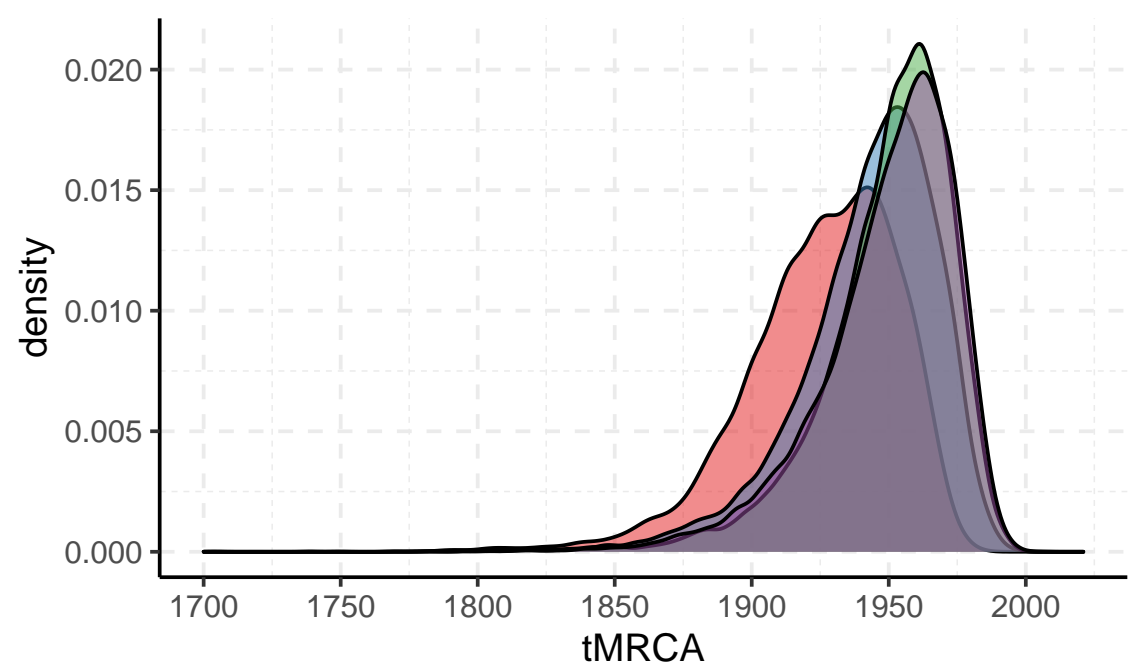

Molecular Dating Method

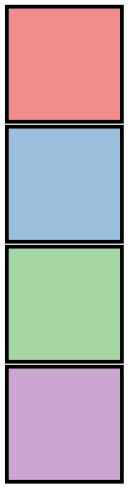

BactDating

tMRCA $=1925.62$ [1873.38-1971.86]

BEAST2 - Coalescent Constant Population

tMRCA = 1942.55 [1891-1983.98]

BEAST2 - Coalescent Exponential Population tMRCA = 1949.43 [1901.82-1987.71]

BEAST2 - Coalescent Bayesian Skyline tMRCA = 1949.62 [1899.73-1988.67]

\section{Tn3000}

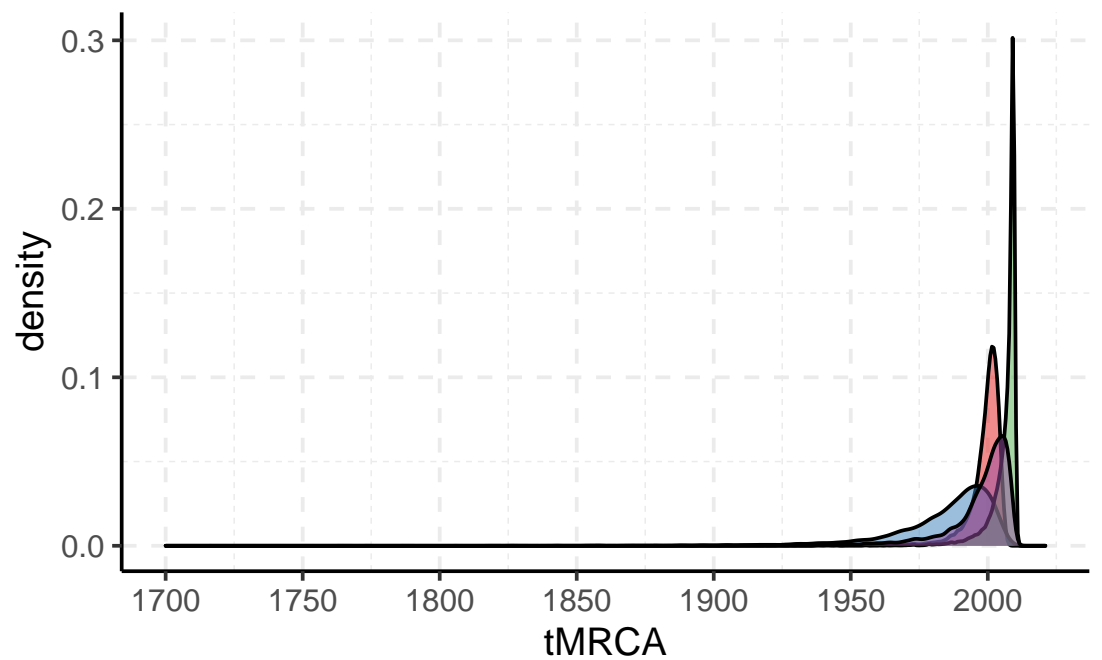

\section{Molecular Dating Method}

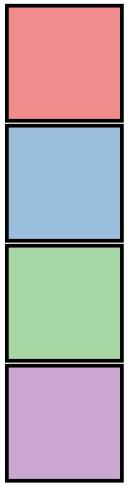

BactDating

tMRCA = 1998.63 [1986.65-2006.32]

BEAST2 - Coalescent Constant Population tMRCA = 1983.94 [1945.78-2007.71]

BEAST2 - Coalescent Exponential Population tMRCA $=2006.15$ [1996.03-2009.5]

BEAST2 - Coalescent Bayesian Skyline tMRCA = 1996.11 [1966.85-2009.49] 


\section{(bosition (bp)}

Proportion of Samples CutA gros $\longrightarrow$ groL $\longrightarrow$ otnC $\longrightarrow$ ISABba125 $\rightarrow$ blaNDM $\rightarrow$ ble $\rightarrow$ trpp $\rightarrow$ dsbD cutA gros $\longrightarrow$ groL $\longrightarrow \longrightarrow$ 1128 blaNDM - ble - trpF $\rightarrow$ dsbD cutA gros $\longrightarrow$ grol $\longrightarrow$

118 blaNDM ble $\rightarrow$ trpF $\rightarrow$ dsbD cutA gros $\longrightarrow$ grol $\longrightarrow$ wapA $\longrightarrow$ 158963

213

thin
blaNDM $\rightarrow$ ble $\rightarrow$ trpF $\rightarrow$ dsbD

cutA gros $\longrightarrow$ grol $\longrightarrow-$ ISCR27

$\rightarrow$ CUtA gros $\rightarrow$ GSCR27 $1999 \rightarrow 96$ blaNDM ble $\rightarrow$ trpF $\rightarrow$ dsbD

$192 \quad \begin{array}{r}96 \\ 35\end{array}$ 2359 1

$3966-$

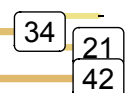

28132

281

5539

85

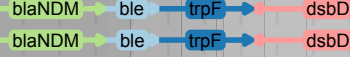
blaNDM $\rightarrow$ ble $\longrightarrow \mathrm{trpF} \rightarrow$ dsbD cutA gros $\longrightarrow$ grol $\longrightarrow$ hyp. ORF $<$ hin - yafQ blaNDM - ble $\rightarrow$ trpF $\rightarrow$ dsbD blaNDM - ble $\rightarrow$ trpF $\rightarrow$ ds $\rightarrow$

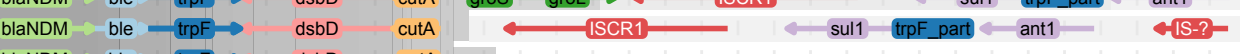

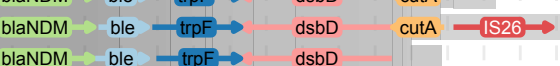
blanDM - ble $\rightarrow$ trpF $\rightarrow$ dsbD blanDM $\rightarrow \quad-$ trpF $\rightarrow$ blaNDM $\rightarrow$ ble $\rightarrow$ trpF $\rightarrow$ dsbD

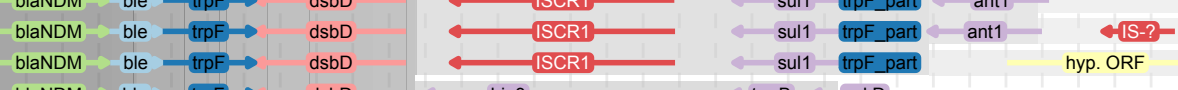
blaNDM - ble $\rightarrow$ trpF $\rightarrow$ dsbD $\rightarrow$ bin3 $\quad$ tmrB yokD blaNDM $\rightarrow$ ble $\rightarrow$ troF $\rightarrow$ dsbD

$$
\text { hyp. ORF tnpR- }
$$
blaNDM ble - trpF--dsbD

- . 65 blandM - ble $\rightarrow$ trpF $\rightarrow$
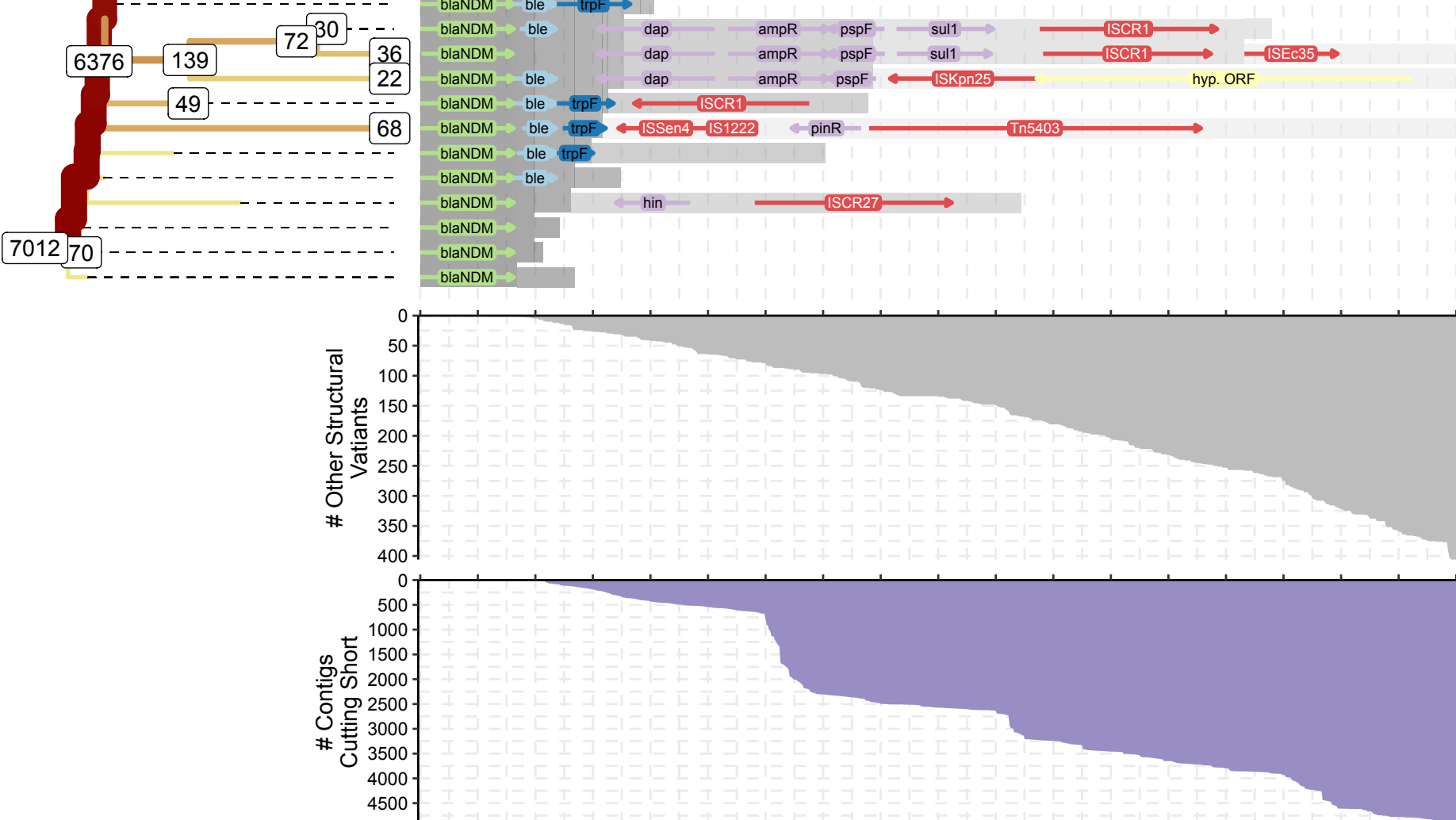

ISCR1-

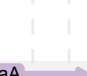

\# Samples blaNDM ble $\rightarrow$ trpF $\rightarrow$ dsbD

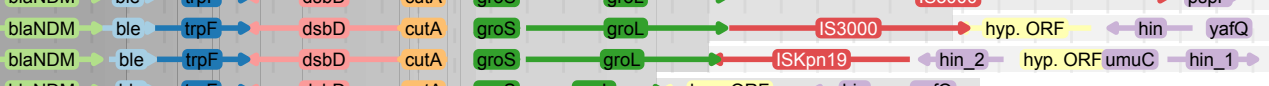
dsbD cutA
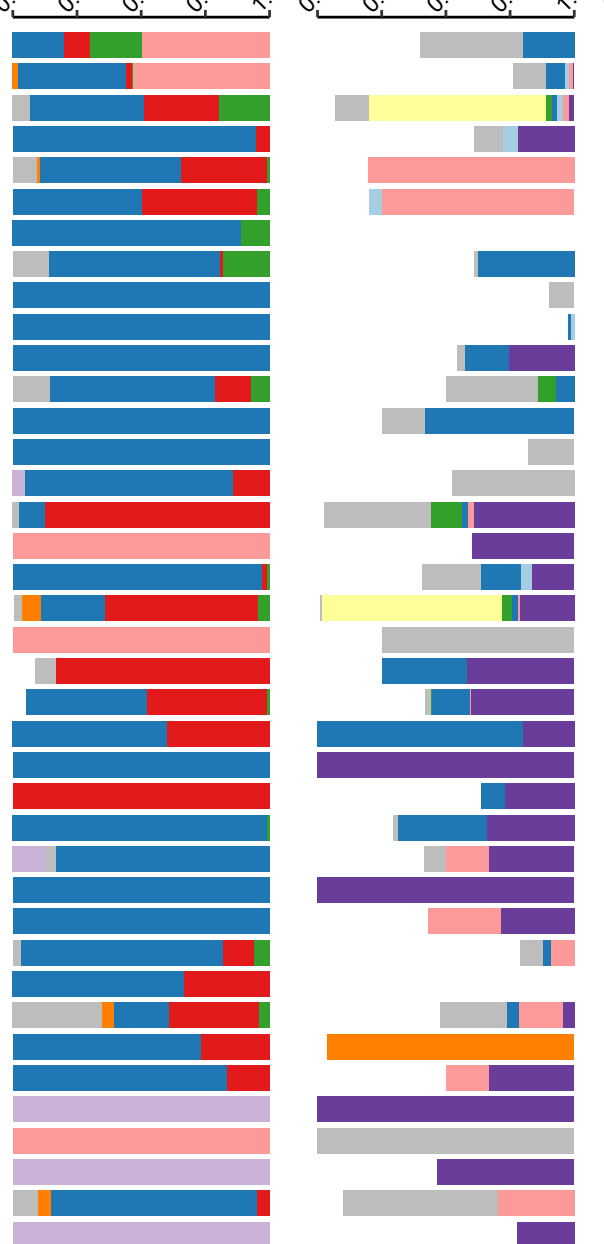

Genus

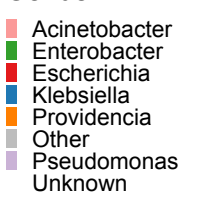

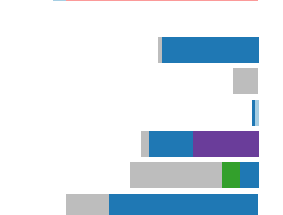

(i)
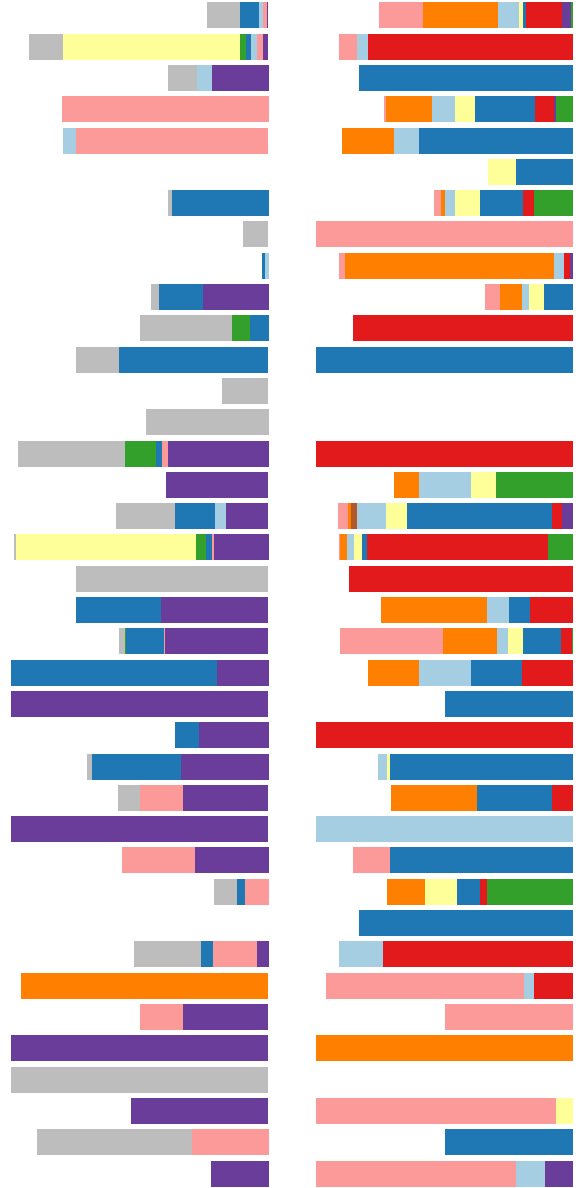

Plasmid Type

$<10 \mathrm{~kb}$ plasmids

IncC $/$ IncFIncHI1B

IncFl/FI

$\mathrm{IncH} 22$
IncN2
Inc 23

Other plasmids
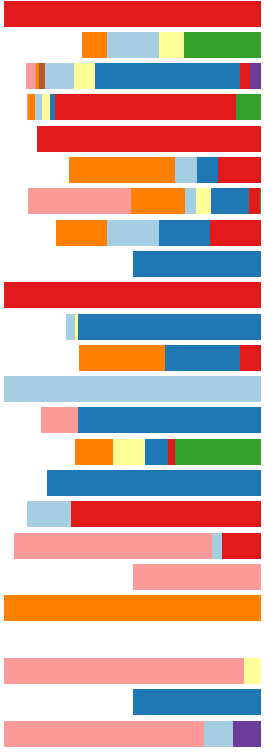

Region

Africa

East Asia

Middle East $\&$ Narth

North America
Oceania

South Asia
South East Asia
Unknown

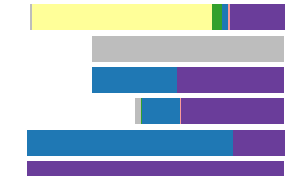

Undefined sequences 